\title{
A UNIFIED CONVERGENCE ANALYSIS FOR LOCAL PROJECTION STABILISATIONS APPLIED TO THE OSEEN PROBLEM*
}

\author{
Gunar Matthies $^{1}$, Piotr Skrzypacz ${ }^{2}$ and Lutz Tobiska ${ }^{2}$
}

\begin{abstract}
The discretisation of the Oseen problem by finite element methods may suffer in general from two shortcomings. First, the discrete inf-sup (Babuška-Brezzi) condition can be violated. Second, spurious oscillations occur due to the dominating convection. One way to overcome both difficulties is the use of local projection techniques. Studying the local projection method in an abstract setting, we show that the fulfilment of a local inf-sup condition between approximation and projection spaces allows to construct an interpolation with additional orthogonality properties. Based on this special interpolation, optimal a-priori error estimates are shown with error constants independent of the Reynolds number. Applying the general theory, we extend the results of Braack and Burman for the standard two-level version of the local projection stabilisation to discretisations of arbitrary order on simplices, quadrilaterals, and hexahedra. Moreover, our general theory allows to derive a novel class of local projection stabilisation by enrichment of the approximation spaces. This class of stabilised schemes uses approximation and projection spaces defined on the same mesh and leads to much more compact stencils than in the two-level approach. Finally, on simplices, the spectral equivalence of the stabilising terms of the local projection method and the subgrid modelling introduced by Guermond is shown. This clarifies the relation of the local projection stabilisation to the variational multiscale approach.
\end{abstract}

Mathematics Subject Classification. 65N12, 65N30, 76D05.

Received October 6, 2006. Revised February 20, 2007.

\section{INTRODUCTION}

The discretisation of the Oseen problem by finite element methods may suffer in general from two shortcomings. First, the discrete inf-sup (Babuška-Brezzi) condition can be violated. Second, spurious oscillations occur in case of higher Reynolds numbers due to the dominating convection. The idea of streamline upwind Petrov-Galerkin (SUPG) stabilisation has been proposed for the advective term in [12] and extended to the Stokes equations in [27] where a pressure stabilisation Petrov-Galerkin (PSPG) method is considered accommodating low equal-order interpolation to be stable and convergent. This formulation circumvented the need to satisfy the discrete inf-sup condition for many interpolations. In an attempt to get the stability features of these works, a method is proposed in [16] that is at the same time advective stable and overcomes the inf-sup

Keywords and phrases. Stabilised finite elements, Navier-Stokes equations, equal-order interpolation.

* Partially supported by the German Research Foundation (DFG) through grants To143 and FOR 447.

1 Fakultät für Mathematik, Ruhr-Universität Bochum, Universitätsstraße 150, 44780 Bochum, Germany. gunar.matthies@ruhr-uni-bochum.de

2 Institut für Analysis und Numerik, Otto-von-Guericke-Universität Magdeburg, Postfach 4120, 39016 Magdeburg, Germany. piotr.skrzypacz@mathematik.uni-magdeburg.de; tobiska@mathematik.uni-magdeburg.de 
restrictions of the standard Galerkin method. A detailed error analysis of these SUPG/PSPG-type stabilisations applied to the incompressible Navier-Stokes equations, including both the case of inf-sup stable and equal-order interpolations, can be found in [41]. Despite the progress of the SUPG/PSPG method in theory and application, an essential drawback of this method is - in particular for higher order interpolations - that various terms need to be added to the weak formulation to guarantee the consistency of the method in a strong way (Galerkin orthogonality holds for smooth solutions). Residual-based stabilisation methods which use inf-sup stable pairs of elements reduce the number of terms which have to be added to the Galerkin formulation [17,33]. However, an additional coupling term between velocity and pressure makes their analysis difficult. Over the last years, several approaches have been developed to relax the strong coupling of velocity and pressure in SUPG/PSPG-type stabilisations and to introduce symmetric versions of the stabilising terms, for an overview see $[11,32]$.

The local projection method is designed for equal-order interpolation and allows a separation of velocity and pressure in the stabilisation terms. It has been introduced for the Stokes problem in [4], extended to the transport equation in [5], and analysed for low order discretisations of the Oseen equations in [7]. Some variants and applications are discussed in $[8,9]$. In the local projection method, the stabilisation term is based on a projection $\pi_{h}: Y_{h} \rightarrow D_{h}$ of the finite element space $Y_{h}$ approximating velocity and pressure into a discontinuous space $D_{h}$. Stabilisation of the standard Galerkin method is achieved by adding terms which give a weighted $L^{2}$-control over the fluctuations $\left(i d-\pi_{h}\right)$ of the gradients of the quantity of interest. The key idea in the error analysis of the local projection scheme is the construction of an interpolant into $Y_{h}$ which exhibits an additional orthogonality property with respect to the discontinuous space $D_{h}$. In [7], the case of low order $Q_{r}$-elements $(r=1,2)$ on quadrilaterals $(d=2)$ and hexahedra $(d=3)$ has been considered. There, $\pi_{h}$ has been chosen to be the $L^{2}$-projection onto the space of discontinuous $Q_{r-1}$-elements on a coarser mesh. Unfortunately, this two-level approach leads to a stencil being less compact than for the SUPG/PSPG-type stabilisation.

The main objective of this paper is to give a general convergence theory of local projection schemes leading to a-priori error estimates which show the same optimal order of convergence as known for the SUPG/PSPG method. To this end, we study under which conditions an interpolant into $Y_{h}$ with additional orthogonality properties with respect to $D_{h}$ can be constructed. We show that an inf-sup condition for the spaces $Y_{h}$ and $D_{h}$ is sufficient for the existence of such an interpolant. Our general theory allows us to consider large classes of spaces $Y_{h}$ and $D_{h}$, including the two-level approach on simplices, quadrilaterals, and hexahedra for arbitrary but fixed polynomial degree $r \geq 1$. Moreover, we can derive local projection schemes not only as a two-level approach but also for pairs of spaces $Y_{h} / D_{h}$ which are defined on the same mesh family $\mathcal{T}_{h}$. This opens the way to circumvent the disadvantage of the classical two-level form of the local projection scheme which produces a larger stencil. As we will show, this new approach of enriched approximation spaces works also on simplices, quadrilaterals, and hexahedra for arbitrary polynomial degree $r \geq 1$.

It is well known that stabilised methods can also be derived from a variational multiscale formulation $[24-26,40]$. Based on a scale separation of the underlying finite element spaces, it has been shown that it is sufficient to stabilise only the fine scale fluctuations. This results into a stabilising term which gives a weighted $L^{2}$-control over the gradient of fluctuations instead of the fluctuations of gradients $[15,19]$. We will discuss the relation between this subgrid modelling approach and the local projection scheme in detail. In particular, we show that for linear elements on simplices both approaches lead to the same discrete problem.

The reader will notice that the idea of local projection results in a large number of concrete schemes. They differ in the specification of the solution and projection space $Y_{h}$ and $D_{h}$, respectively, in the way of satisfying the local inf-sup condition (by a two-level approach or by taking enrichments), and in considering fluctuations of the gradients or gradients of fluctuations. Thus, the evaluation of the full potential of each of these methods needs a thorough numerical study which is beyond the scope of this paper. In the following we refer to numerical results obtained by schemes belonging to the considered class of stabilisation methods. The application of subgrid modelling (gradients of fluctuations) to scalar transport equations of convection-diffusion type has been numerically studied in $[15,19-23]$ in the two-level context for continous piecewise linear and quadratic elements. 
The extension to nonconfoming piecewise linear elements enriched by continuous cubic or nonconforming quadratic bubble functions has been considered in [1]. It is remarkable that the local projection stabilisation is also useful for control problems of convection-diffusion type [6]. In [30], the projection of the gradients onto piecewise constants on a coarser mesh for linear and bilinear elements have been studied. Numerical tests showing the evidence of the pressure stabilisation of equal order interpolation for the Stokes problem are presented in [4]. Continuous, piecewise bilinears and biquadratics have been studied there in a two-level context. Stabilisation of both phenomena, dominating convection and violation of the discrete Babuška-Brezzi condition, are investigated in [7-9,23] for the incompressible Navier-Stokes equation. For the flow around a NACA 0012 airfoil, numerical results are given for Reynolds numbers up to $10^{6}$ in [23]. Convincing are also the result of a benchmark problem of the flow around a cylinder in 2D [9] and 3D [8]. In these papers, a two-level method based on quadratic quadrilateral and hexahedral elements with hanging nodes has been used. In [10], the method is extended to compressible Navier-Stokes equations coupled with further transport equations to describe chemically reacting flows. Finally, turbulent flows including the 3D mixing layer problem are numerically computed by the local projection method in $[29,31]$.

The plan of the paper is as follows. In Section 1, a weak formulation of the Oseen equations and its standard Galerkin discretisation is given. We formulate the local projection method in an abstract setting. Section 2 is devoted to the convergence analysis of the local projection method in this abstract setting. The basic tool is the construction of a special interpolant based on the fulfilment of a local inf-sup condition. Proving the stability independent of the Reynolds number and the approximated Galerkin orthogonality, we conclude optimal a-priori estimates. The application of the theory in the framework of two-level methods is studied in Section 3 where the focus is on defining pairs of finite element spaces satisfying the local inf-sup condition given in Section 2. We extend in Section 4 the analysis to spaces which are defined on the same mesh. Starting from the space $D_{h}$, the space $Y_{h}$ is obtained by enriching standard finite element spaces. Additionally, we study the relation of local projection schemes to the subgrid modelling approach in Section 5. We summarise our results in Section 6.

Notation. Throughout the paper $C$ will denote a generic positive constant which is independent of the Reynolds number and the mesh. Subscripted constants such as $C_{1}$ are also independent of the Reynolds number and the mesh, but have a fixed value. We will write shortly $\alpha \sim \beta$, if there are positive constants $\underline{C}$ and $\bar{C}$ such that

$$
\underline{C} \beta \leq \alpha \leq \bar{C} \beta
$$

holds.

Our Oseen problem will be considered in the domain $\Omega \subset \mathbb{R}^{d}, d=2,3$, which is assumed to be a polygonal or polyhedral domain with boundary $\partial \Omega$. For a measurable subset $G$ of $\Omega$, the usual Sobolev spaces $W^{m, p}(G)$ with norm $\|\cdot\|_{m, p, G}$ and semi-norm $|\cdot|_{m, p, G}$ are used. In the case $p=2$, we have $H^{m}(G)=W^{m, 2}(G)$ and the index $p$ will be omitted. The $L^{2}$ inner product on $G$ is denoted by $(\cdot, \cdot)_{G}$. Note that the index $G$ will be omitted for $G=\Omega$. This notation of norms, semi-norms, and inner products is also used for the vector-valued and tensor-valued case.

\section{LOCAL PROJECTION STABILISATION IN AN ABSTRACT SETTING}

\subsection{Weak formulation of the Oseen problem}

Let us consider the Oseen problem

$$
-\nu \Delta \mathbf{u}+(\mathbf{b} \cdot \nabla) \mathbf{u}+\sigma \mathbf{u}+\nabla p=\mathbf{f} \quad \text { in } \Omega, \quad \nabla \cdot \mathbf{u}=0 \quad \text { in } \Omega, \quad \mathbf{u}=0 \quad \text { on } \partial \Omega
$$

as a linearisation of the steady $(\sigma=0)$ and the non-steady $(\sigma>0)$ time-discretised Navier-Stokes equations. Here, we assume $\mathbf{b} \in \mathbf{W}^{1, \infty}(\Omega)$ with $\nabla \cdot \mathbf{b}=0$. Let $\mathbf{V}=\mathbf{H}_{0}^{1}(\Omega), Q=L_{0}^{2}(\Omega)=\left\{q \in L^{2}(\Omega):(q, 1)=0\right\}$. 
We introduce on the product space $\mathbf{V} \times Q$ the bilinear form $A$ given by

$$
A((\mathbf{u}, p) ;(\mathbf{v}, q)):=\nu(\nabla \mathbf{u}, \nabla \mathbf{v})+((\mathbf{b} \cdot \nabla) \mathbf{u}, \mathbf{v})+\sigma(\mathbf{u}, \mathbf{v})-(p, \nabla \cdot \mathbf{v})+(q, \nabla \cdot \mathbf{u})
$$

A weak formulation of the Oseen problem (1.1) reads:

$$
\text { Find }(\mathbf{u}, p) \in \mathbf{V} \times Q \text { such that for all }(\mathbf{v}, q) \in \mathbf{V} \times Q: \quad A((\mathbf{u}, p) ;(\mathbf{v}, q))=(\mathbf{f}, \mathbf{v}) \text {. }
$$

The property

$$
((\mathbf{b} \cdot \nabla) \mathbf{v}, \mathbf{v})=\frac{1}{2}(\mathbf{b} \cdot \nabla(\mathbf{v} \cdot \mathbf{v}), 1)=-\frac{1}{2}(\nabla \cdot \mathbf{b}, \mathbf{v} \cdot \mathbf{v})=0 \quad \forall \mathbf{v} \in \mathbf{V}
$$

allows to apply the Lax-Milgram lemma in the subspace of divergence-free functions and to establish a unique velocity field $\mathbf{u}$. A unique pressure $p \in Q$ such that $(\mathbf{u}, p)$ solves (1.3) follows from the Babuška-Brezzi condition for the pair $(\mathbf{V}, Q)[18]$.

\subsection{Galerkin discretisation}

For the finite element discretisation of the Oseen problem (1.3), we introduce a shape regular decomposition $\mathcal{T}_{h}$ of $\Omega$ into $d$-dimensional simplices, quadrilaterals or hexahedra. The diameter of a cell $K$ will be denoted by $h_{K}$ and the mesh parameter $h$ represents the maximum diameter of the cells $K \in \mathcal{T}_{h}$. Let $Y_{h} \subset H^{1}(\Omega)$ be a finite element space of continuous, piecewise polynomial functions defined over $\mathcal{T}_{h}$.

Assumption A1: There is an interpolation operator $i_{h}: H^{1}(\Omega) \rightarrow Y_{h}$ such that $i_{h}: H_{0}^{1}(\Omega) \rightarrow Y_{h} \cap H_{0}^{1}(\Omega)$ and

$$
\left\|w-i_{h} w\right\|_{0, K}+h_{K}\left|w-i_{h} w\right|_{1, K} \leq C h_{K}^{l}\|w\|_{l, \omega(K)} \quad \forall w \in H^{l}(\omega(K)), \forall K \in \mathcal{T}_{h}, 1 \leq l \leq r+1
$$

where $\omega(K)$ denotes a certain local neighbourhood of $K$ which appears in the definition of these interpolation operators for non-smooth functions, see $[14,38]$ for more details.

We will also apply this type of interpolation operator to vector-valued functions in a component-wise manner. We indicate this by using boldface notations, for example $\mathbf{i}_{h}: \mathbf{V} \rightarrow Y_{h}^{d} \cap \mathbf{V}$.

Remark 1.1. The existence theory of interpolation operators for non-smooth functions satisfying Assumption A1 is well established in the literature, see $[2,14,38]$.

For simplicity of presentation, we consider in this paper the case of equal-order interpolation, thus assuming $\mathbf{V}_{h}:=Y_{h}^{d} \cap \mathbf{V}$ and $Q_{h}:=Y_{h} \cap Q$. Now, the standard Galerkin discretisation of (1.3) reads:

$$
\text { Find }\left(\mathbf{u}_{h}, p_{h}\right) \in \mathbf{V}_{h} \times Q_{h} \text { such that for all }\left(\mathbf{v}_{h}, q_{h}\right) \in \mathbf{V}_{h} \times Q_{h}: A\left(\left(\mathbf{u}_{h}, p_{h}\right) ;\left(\mathbf{v}_{h}, q_{h}\right)\right)=\left(\mathbf{f}, \mathbf{v}_{h}\right) \text {. }
$$

In general, this formulation suffers from two reasons: the violation of the discrete Babuška-Brezzi condition

$$
\exists \beta_{0}>0: \inf _{q_{h} \in Q_{h}} \sup _{\mathbf{v}_{h} \in \mathbf{V}_{h}} \frac{\left(q_{h}, \nabla \cdot \mathbf{v}_{h}\right)}{\left\|q_{h}\right\|_{0}\left|\mathbf{v}_{h}\right|_{1}} \geq \beta_{0},
$$

and the dominating advection in case of $\nu \ll 1$. Both instability phenomena can be handled by the local projection technique which will be the topic of the next subsection. 


\subsection{Stabilisation by local projection}

In order to explain the stabilisation method, we start with some additional notations. By a macro element $M$ we denote the union of one or more neighbouring cells $K \in \mathcal{T}_{h}$. The local neighbourhood $\omega(K)$ introduced in the

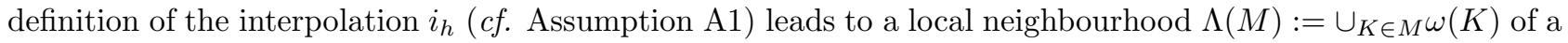
macro element $M$. The diameter of a macro element $M$ is denoted by $h_{M}$. We assume that the decomposition of $\Omega$ into macro elements $M \in \mathcal{M}_{h}$ is non-overlapping and also shape regular, moreover

$$
h_{K} \sim h_{M}, \quad \forall K \subset M, \quad \forall M \in \mathcal{M}_{h}
$$

One can think of having first the decomposition $\mathcal{M}_{h}$ into macro elements from which the decomposition $\mathcal{T}_{h}$ is generated by certain refinement rules. Note that we also allow the case $\mathcal{M}_{h}=\mathcal{T}_{h}$.

Let $D_{h}$ denote a discontinuous finite element space defined on the macro decomposition $\mathcal{M}_{h}$ and $D_{h}(M):=$ $\left\{\left.q_{h}\right|_{M}: q_{h} \in D_{h}\right\}$. Further, let $\pi_{M}: L^{2}(M) \rightarrow D_{h}(M)$ be a local projection which defines the projection $\pi_{h}: L^{2}(\Omega) \rightarrow D_{h}$ by $\left.\left(\pi_{h} w\right)\right|_{M}:=\pi_{M}\left(\left.w\right|_{M}\right)$. Associated with the projection $\pi_{h}$ is the fluctuation operator $\kappa_{h}: L^{2}(\Omega) \rightarrow L^{2}(\Omega)$ defined by $\kappa_{h}:=i d-\pi_{h}$, where $i d: L^{2}(\Omega) \rightarrow L^{2}(\Omega)$ is the identity. As in the previous subsection, we apply these operators to vector-valued functions in a component-wise manner and indicate this by using boldface notations, e.g. $\boldsymbol{\pi}_{h}: \mathbf{L}^{2}(\Omega) \rightarrow \mathbf{D}_{h}$ and $\boldsymbol{\kappa}_{h}: \mathbf{L}^{2}(\Omega) \rightarrow \mathbf{L}^{2}(\Omega)$.

Assumption A2: Let the fluctuation operator $\kappa_{h}$ satisfy the following approximation property:

$$
\left\|\kappa_{h} q\right\|_{0, M} \leq C h_{M}^{l}|q|_{l, M} \quad \forall q \in H^{l}(M), \forall M \in \mathcal{M}_{h}, 0 \leq l \leq r .
$$

Remark 1.2. We shortly discuss the case in which $\pi_{h}$ is the $L^{2}$-projection in $D_{h}$ and the space $D_{h}(M)$ contains the space $P_{r-1}(M)$ of polynomials of degree less than or equal to $r-1, r \geq 1$. This means that

$$
\left(\pi_{h} w-w, w_{h}\right)=0 \quad \forall w_{h} \in D_{h}, w \in L^{2}(\Omega),
$$

and

$$
\bigoplus_{M \in \mathcal{M}_{h}} P_{r-1}(M) \subset D_{h}
$$

Since $D_{h}$ is discontinuous over the macro element faces, (1.9) can be localised and $\pi_{M}: L^{2}(M) \rightarrow D_{h}(M)$ is locally defined by

$$
\left(\pi_{M} w-w, w_{h}\right)_{M}=0 \quad \forall w_{h} \in D_{h}(M), w \in L^{2}(M)
$$

In this case, the $L^{2}$-projection $\pi_{M}: L^{2}(M) \rightarrow D_{h}(M)$ becomes the identity on the subspace $P_{r-1}(M) \subset H^{l}(M)$. Now, the Bramble-Hilbert lemma gives the approximation properties for $\kappa_{h}=i d-\pi_{h}$ stated in Assumption A2.

We will modify the discrete problem (1.6) by adding the stabilisation term

$$
\begin{aligned}
S_{h}\left(\left(\mathbf{u}_{h}, p_{h}\right) ;\left(\mathbf{v}_{h}, q_{h}\right)\right):= & \sum_{M \in \mathcal{M}_{h}}\left(\tau_{M}\left(\boldsymbol{\kappa}_{h}(\mathbf{b} \cdot \nabla) \mathbf{u}_{h}, \boldsymbol{\kappa}_{h}(\mathbf{b} \cdot \nabla) \mathbf{v}_{h}\right)_{M}\right. \\
& \left.+\mu_{M}\left(\kappa_{h} \nabla \cdot \mathbf{u}_{h}, \kappa_{h} \nabla \cdot \mathbf{v}_{h}\right)_{M}+\alpha_{M}\left(\boldsymbol{\kappa}_{h} \nabla p_{h}, \boldsymbol{\kappa}_{h} \nabla q_{h}\right)_{M}\right)
\end{aligned}
$$

where $\tau_{M}, \mu_{M}$, and $\alpha_{M}$ are user-chosen constants. Their optimal mesh-dependent choice will follow from the error analysis of the method. Now, our stabilised scheme reads: 
Find $\left(\mathbf{u}_{h}, p_{h}\right) \in \mathbf{V}_{h} \times Q_{h}$ such that for all $\left(\mathbf{v}_{h}, q_{h}\right) \in \mathbf{V}_{h} \times Q_{h}$ :

$$
A\left(\left(\mathbf{u}_{h}, p_{h}\right) ;\left(\mathbf{v}_{h}, q_{h}\right)\right)+S_{h}\left(\left(\mathbf{u}_{h}, p\right) ;\left(\mathbf{v}_{h}, q_{h}\right)\right)=\left(\mathbf{f}, \mathbf{v}_{h}\right) .
$$

Existence, uniqueness, and convergence properties of the solutions $\left(\mathbf{u}_{h}, p_{h}\right) \in \mathbf{V}_{h} \times Q_{h}$ will be studied in the next section.

\section{Convergence Analysis}

\subsection{Special interpolant}

The key ingredient of the error analysis of the local projection method is the construction of an interpolant $j_{h}: H^{1}(\Omega) \rightarrow Y_{h}$ such that the error $w-j_{h} w$ is $L^{2}$-orthogonal to $D_{h}$ without loosing the standard approximation properties. Let $Y_{h}(M):=\left\{\left.w_{h}\right|_{M}: w_{h} \in Y_{h}, w_{h}=0\right.$ on $\left.\Omega \backslash M\right\}$.

Assumption A3: Let the local inf-sup condition

$$
\exists \beta_{1}>0, \quad \forall h>0 \forall M \in \mathcal{M}_{h}: \quad \inf _{q_{h} \in D_{h}(M)} \sup _{v_{h} \in Y_{h}(M)} \frac{\left(v_{h}, q_{h}\right)_{M}}{\left\|v_{h}\right\|_{0, M}\left\|q_{h}\right\|_{0, M}} \geq \beta_{1}>0
$$

be satisfied.

Remark 2.1. It is clear that $Y_{h}(M)$ - compared to $D_{h}(M)$ - has to be rich enough for satisfying A3. In particular, a necessary requirement is

$$
\operatorname{dim} Y_{h}(M) \geq \operatorname{dim} D_{h}(M) .
$$

On the other hand $D_{h}$ has to be large enough to guarantee A2. In Section 3, we follow this idea for a given space $Y_{h}(M)$ by choosing $D_{h}$ as a discontinuous finite element space on a coarser mesh; its dimension small enough to satisfy A3 but big enough to fulfil A2. A different strategy is used in Section 4 where both spaces are defined on the same mesh, $D_{h}(M)$ such that A2 holds and $Y_{h}(M)$ is enriched by additional functions to fulfil A3.

Theorem 2.2. Let Assumptions $\mathrm{A} 1$ and $\mathrm{A} 3$ be satisfied. Then, there are interpolation operators $j_{h}: H^{1}(\Omega) \rightarrow$ $Y_{h}$ and $\mathbf{j}_{h}: \mathbf{V} \rightarrow \mathbf{V}_{h}$ satisfying the following orthogonality and approximation properties:

$$
\begin{array}{rlrl}
\left(w-j_{h} w, q_{h}\right) & =0 & & \forall q_{h} \in D_{h}, \forall w \in H^{1}(\Omega), \\
\left\|w-j_{h} w\right\|_{0, M}+h_{M}\left|w-j_{h} w\right|_{1, M} \leq C h_{M}^{l}\|w\|_{l, \Lambda(M)} & & \forall w \in H^{l}(\Omega), 1 \leq l \leq r+1, \forall M \in \mathcal{M}_{h} \\
\left(\mathbf{w}-\mathbf{j}_{h} \mathbf{w}, \mathbf{q}_{h}\right)=0 & & \forall \mathbf{q}_{h} \in \mathbf{D}_{h}, \forall \mathbf{w} \in \mathbf{V}, \\
\left\|\mathbf{w}-\mathbf{j}_{h} \mathbf{w}\right\|_{0, M}+h_{M}\left|\mathbf{w}-\mathbf{j}_{h} \mathbf{w}\right|_{1, M} \leq C h_{M}^{l}\|\mathbf{w}\|_{l, \Lambda(M)} & & \forall \mathbf{w} \in \mathbf{V} \cap \mathbf{H}^{l}(\Omega), 1 \leq l \leq r+1, \forall M \in \mathcal{M}_{h} .
\end{array}
$$

Proof. We use a result in [18], Lemma I.4.1: the linear continuous operator $B_{h}: Y_{h}(M) \rightarrow D_{h}(M)^{\prime}$ defined by

$$
\left\langle B_{h} v_{h}, q_{h}\right\rangle_{D_{h}(M)}:=\left(v_{h}, q_{h}\right)_{M} \quad \forall v_{h} \in Y_{h}(M), q_{h} \in D_{h}(M)
$$

is an isomorphism from $W_{h}(M)^{\perp}$ onto $D_{h}(M)^{\prime}$ with

$$
\beta_{1}\left\|v_{h}\right\|_{0, M} \leq\left\|B_{h} v_{h}\right\|_{D_{h}(M)^{\prime}} \quad \forall v_{h} \in W_{h}(M)^{\perp}
$$

if and only if A3 holds. Here, $D_{h}(M)^{\prime}$ denotes the dual space of $D_{h}(M)$,

$$
W_{h}(M):=\left\{v_{h} \in Y_{h}(M):\left(v_{h}, q_{h}\right)=0 \forall q_{h} \in D_{h}(M)\right\},
$$


and $W_{h}(M)^{\perp}$ the $L^{2}$-orthogonal complement of $W_{h}(M)$ in $Y_{h}(M)$. Consequently, for each $w \in H^{1}(\Omega)$ we have a unique $z_{h}(w) \in W_{h}(M)^{\perp}$ such that

$$
\begin{aligned}
\left\langle B_{h} z_{h}(w), q_{h}\right\rangle_{D_{h}(M)} & =\left(z_{h}(w), q_{h}\right)_{M}=\left(w-i_{h} w, q_{h}\right)_{M} \quad \forall q_{h} \in D_{h}(M), \\
\left\|z_{h}(w)\right\|_{0, M} & \leq \frac{1}{\beta_{1}}\left\|w-i_{h} w\right\|_{0, M} .
\end{aligned}
$$

We take $\left.j_{h} w\right|_{M}:=\left.i_{h} w\right|_{M}+z_{h}(w)$ for all $M \in \mathcal{M}_{h}$. According to $\bigoplus_{M \in \mathcal{M}_{h}} Y_{h}(M) \subset Y_{h}$, a global interpolation $j_{h}: H^{1}(\Omega) \rightarrow Y_{h}$ is defined which satisfies for all $M \in \mathcal{M}_{h}$

$$
\left\|w-j_{h} w\right\|_{0, M} \leq\left(1+\frac{1}{\beta_{1}}\right)\left\|w-i_{h} w\right\|_{0, M} \leq C h_{M}^{l}\|w\|_{l, \Lambda(M)} \quad \forall w \in H^{l}(\Omega), 1 \leq l \leq r+1 .
$$

The orthogonality property (2.3) follows from (2.7) and the definition of $j_{h}$. It remains to show the approximation property for the $H^{1}$-seminorm. To this end, we apply an inverse inequality and (2.8) to get

$$
\left|z_{h}(w)\right|_{1, M} \leq C h_{M}^{-1}\left\|z_{h}(w)\right\|_{0, M} \leq C h_{M}^{-1}\left\|w-i_{h} w\right\|_{0, M} .
$$

Using the triangle inequality, (2.10) and the approximation property (1.5), we conclude

$$
\left|w-j_{h} w\right|_{1, M} \leq\left|w-i_{h} w\right|_{1, M}+\left|z_{h}(w)\right|_{1, M} \leq C h_{M}^{l-1}\|w\|_{l, \Lambda(M)} .
$$

Now, (2.5) and (2.6) are extensions to the vector-valued case. Taking into consideration the definition $\mathbf{j}_{h} \mathbf{w}=\mathbf{i}_{h} \mathbf{w}+\mathbf{z}_{h}(w)$, the mapping property $\mathbf{i}_{h}: \mathbf{V} \rightarrow \mathbf{V}_{h}$, and that $\mathbf{z}_{h}(\mathbf{w})$ vanishes on the boundary $\partial \Omega$, we get $\mathbf{j}_{h}: \mathbf{V} \rightarrow \mathbf{V}_{h},(2.5)$, and (2.6).

Remark 2.3. We see from (2.9) that the constant $\beta_{1}$ should be independent of the mesh size $h$ to guarantee the approximation properties (2.4) and (2.6) of the interpolant $j_{h}$. Note that (2.2) does not guarantee that the constant $\beta_{1}$ is independent of the mesh size $h$.

Remark 2.4. Note that by setting $q_{h}=1$ in $(2.3)$ we get $\left(j_{h} w, 1\right)=(w, 1)$ for all $w \in H^{1}(\Omega)$. This implies in particular $j_{h}: H^{1}(\Omega) \cap Q \rightarrow Q_{h}$.

Remark 2.5. Following the ideas in [39] and assuming a family of macro elements which is equivalent to a reference macro element, one can show that (2.1) reduces to show that

$$
N_{M}:=\left\{q_{h} \in D_{h}(M):\left(q_{h}, v_{h}\right)_{M}=0 \quad \forall v_{h} \in V_{h}(M)\right\}=\{0\}
$$

holds true.

\subsection{Stability}

Let us introduce the mesh-dependent norm on the product space $\mathbf{V} \times Q$ by

$$
\|\mid(\mathbf{v}, q)\| \|:=\left(\nu|\mathbf{v}|_{1}^{2}+\sigma\|\mathbf{v}\|_{0}^{2}+(\nu+\sigma)\|q\|_{0}^{2}+S((\mathbf{v}, q) ;(\mathbf{v}, q))\right)^{1 / 2} .
$$

We show that the bilinear form $\left(A+S_{h}\right)$ satisfies an inf-sup condition on $\mathbf{V}_{h} \times Q_{h}$. 
Lemma 2.6. Assume $\mathrm{A} 1, \mathrm{~A} 3$, and $\max \left(\nu, \sigma, \tau_{M}, \mu_{M}, h_{M}^{2} / \alpha_{M}\right) \leq C$ for all $M \in \mathcal{M}_{h}$. Then, there is a positive constant $\beta_{2}$ independent of $\nu$ and $h$ such that

$$
\inf _{\left(\mathbf{v}_{h}, q_{h}\right) \in \mathbf{V}_{h} \times Q_{h}} \sup _{\left(\mathbf{w}_{h}, r_{h}\right) \in \mathbf{V}_{h} \times Q_{h}} \frac{\left(A+S_{h}\right)\left(\left(\mathbf{v}_{h}, q_{h}\right) ;\left(\mathbf{w}_{h}, r_{h}\right)\right)}{\left\|\left|\left(\mathbf{v}_{h}, q_{h}\right)\right|\right\|||||\left(\mathbf{w}_{h}, r_{h}\right) \| \mid} \geq \beta_{2}>0
$$

holds true.

Proof. Let us consider an arbitrary $\left(\mathbf{v}_{h}, q_{h}\right) \in \mathbf{V}_{h} \times Q_{h}$. Choosing $\left(\mathbf{w}_{h}, r_{h}\right)=\left(\mathbf{v}_{h}, q_{h}\right)$, we have

$$
\left(A+S_{h}\right)\left(\left(\mathbf{v}_{h}, q_{h}\right) ;\left(\mathbf{v}_{h}, q_{h}\right)\right)=\nu\left|\mathbf{v}_{h}\right|_{1}^{2}+\sigma\left\|\mathbf{v}_{h}\right\|_{0}^{2}+S_{h}\left(\left(\mathbf{v}_{h}, q_{h}\right) ;\left(\mathbf{v}_{h}, q_{h}\right)\right)
$$

due to property (1.4).

Now we consider another choice to generate an $L^{2}$-norm control over the pressure. For any $q_{h} \in Q_{h}$, the continuous Babuška-Brezzi condition guarantees the existence of a function $\mathbf{v}_{q_{h}} \in \mathbf{V}$ such that

$$
\left(\nabla \cdot \mathbf{v}_{q_{h}}, q_{h}\right)=-\left(q_{h}, q_{h}\right), \quad\left\|\mathbf{v}_{q_{h}}\right\|_{1} \leq C\left\|q_{h}\right\|_{0} .
$$

We choose $\left(\mathbf{w}_{h}, r_{h}\right)=\left(\mathbf{j}_{h} \mathbf{v}_{q_{h}}, 0\right)$ where $\mathbf{j}_{h}$ is the interpolant of Theorem 2.2 satisfying (2.5) and (2.6). Thus, we obtain

$$
\begin{gathered}
A\left(\left(\mathbf{v}_{h}, q_{h}\right) ;\left(\mathbf{j}_{h} \mathbf{v}_{q_{h}}, 0\right)\right)=\left\|q_{h}\right\|_{0}^{2}-\left(q_{h}, \nabla \cdot\left(\mathbf{j}_{h} \mathbf{v}_{q_{h}}-\mathbf{v}_{q_{h}}\right)\right)+\left((\mathbf{b} \cdot \nabla) \mathbf{v}_{h}, \mathbf{j}_{h} \mathbf{v}_{q_{h}}\right) \\
+\nu\left(\nabla \mathbf{v}_{h}, \nabla \mathbf{j}_{h} \mathbf{v}_{q_{h}}\right)+\sigma\left(\mathbf{v}_{h}, \mathbf{j}_{h} \mathbf{v}_{q_{h}}\right) .
\end{gathered}
$$

We estimate the last four terms on the right hand side. Starting with an integration by parts of the first of them, we get

$$
\begin{aligned}
-\left(q_{h}, \nabla \cdot\left(\mathbf{j}_{h} \mathbf{v}_{q_{h}}-\mathbf{v}_{q_{h}}\right)\right) & =\left(\nabla q_{h},\left(\mathbf{j}_{h} \mathbf{v}_{q_{h}}-\mathbf{v}_{q_{h}}\right)\right)=\left(\boldsymbol{\kappa}_{h} \nabla q_{h},\left(\mathbf{j}_{h} \mathbf{v}_{q_{h}}-\mathbf{v}_{q_{h}}\right)\right), \\
\left|\left(q_{h}, \nabla \cdot\left(\mathbf{j}_{h} \mathbf{v}_{q_{h}}-\mathbf{v}_{q_{h}}\right)\right)\right| & \leq\left(\sum_{M \in \mathcal{M}_{h}} \alpha_{M}\left\|\boldsymbol{\kappa}_{h} \nabla q_{h}\right\|_{0, M}^{2}\right)^{1 / 2}\left(\sum_{M \in \mathcal{M}_{h}} \frac{1}{\alpha_{M}}\left\|\mathbf{j}_{h} \mathbf{v}_{q_{h}}-\mathbf{v}_{q_{h}}\right\|_{0, M}^{2}\right)^{1 / 2} \\
& \leq C\left(S_{h}\left(\left(\mathbf{v}_{h}, q_{h}\right) ;\left(\mathbf{v}_{h}, q_{h}\right)\right)\right)^{1 / 2}\left\|\mathbf{v}_{q_{h}}\right\|_{1} \leq C\left(S_{h}\left(\left(\mathbf{v}_{h}, q_{h}\right) ;\left(\mathbf{v}_{h}, q_{h}\right)\right)\right)^{1 / 2}\left\|q_{h}\right\|_{0} \\
& \leq \frac{\left\|q_{h}\right\|_{0}^{2}}{8}+C S_{h}\left(\left(\mathbf{v}_{h}, q_{h}\right) ;\left(\mathbf{v}_{h}, q_{h}\right)\right) .
\end{aligned}
$$

Integrating by parts the third term in (2.15), using the $H^{1}$ stability of $j_{h}$ which follows from Theorem 2.2, and (2.14), we obtain

$$
\left|\left((\mathbf{b} \cdot \nabla) \mathbf{v}_{h}, \mathbf{j}_{h} \mathbf{v}_{q_{h}}\right)\right|=\left|\left(\mathbf{v}_{h},(\mathbf{b} \cdot \nabla) \mathbf{j}_{h} \mathbf{v}_{q_{h}}\right)\right| \leq C\left\|\mathbf{v}_{h}\right\|_{0}\left|\mathbf{j}_{h} \mathbf{v}_{q_{h}}\right|_{1} \leq \frac{\left\|q_{h}\right\|_{0}^{2}}{8}+C\left\|\mathbf{v}_{h}\right\|_{0}^{2}
$$

For estimating the remaining terms in $(2.15)$, we use $\max (\nu, \sigma) \leq C$ to get

$$
\begin{aligned}
\left|\nu\left(\nabla \mathbf{v}_{h}, \nabla \mathbf{j} h \mathbf{v}_{q_{h}}\right)+\sigma\left(\mathbf{v}_{h}, \mathbf{j}_{h} \mathbf{v}_{q_{h}}\right)\right| & \leq\left(\nu\left|\mathbf{v}_{h}\right|_{1}+\sigma\left\|\mathbf{v}_{h}\right\|_{0}\right)\left\|\mathbf{j}_{h} \mathbf{v}_{q_{h}}\right\|_{1} \leq C\left(\nu^{1 / 2}\left|\mathbf{v}_{h}\right|_{1}+\sigma^{1 / 2}\left\|\mathbf{v}_{h}\right\|_{0}\right)\left\|q_{h}\right\|_{0} \\
& \leq \frac{\left\|q_{h}\right\|_{0}^{2}}{8}+C\left(\nu\left|\mathbf{v}_{h}\right|_{1}^{2}+\sigma\left\|\mathbf{v}_{h}\right\|_{0}^{2}\right) .
\end{aligned}
$$


The Cauchy-Schwarz inequality and the $L^{2}$-stability of $\boldsymbol{\kappa}_{h}$ give

$$
\begin{aligned}
\left|S_{h}\left(\left(\mathbf{v}_{h}, q_{h}\right) ;\left(\mathbf{j}_{h} \mathbf{v}_{q_{h}}, 0\right)\right)\right| & \leq C\left(S_{h}\left(\left(\mathbf{v}_{h}, 0\right) ;\left(\mathbf{v}_{h}, 0\right)\right)\right)^{1 / 2}\left|\mathbf{j}_{h} \mathbf{v}_{q_{h}}\right|_{1} \leq C\left(S_{h}\left(\left(\mathbf{v}_{h}, q_{h}\right) ;\left(\mathbf{v}_{h}, q_{h}\right)\right)\right)^{1 / 2}\left\|q_{h}\right\|_{0} \\
& \leq \frac{\left\|q_{h}\right\|_{0}^{2}}{8}+C S_{h}\left(\left(\mathbf{v}_{h}, q_{h}\right) ;\left(\mathbf{v}_{h}, q_{h}\right)\right)
\end{aligned}
$$

Let

$$
X:=\left(\nu\left|\mathbf{v}_{h}\right|_{1}^{2}+\sigma\left\|\mathbf{v}_{h}\right\|_{0}^{2}+S_{h}\left(\left(\mathbf{v}_{h}, q_{h}\right) ;\left(\mathbf{v}_{h}, q_{h}\right)\right)\right)^{1 / 2}
$$

denote the part of the triple norm without $L^{2}$-control over the pressure. Using (2.16)-(2.19), we get from

$$
\left(A+S_{h}\right)\left(\left(\mathbf{v}_{h}, q_{h}\right) ;\left(\mathbf{j}_{h} \mathbf{v}_{q_{h}}, 0\right)\right) \geq \frac{\left\|q_{h}\right\|_{0}^{2}}{2}-C X^{2}-C\left\|\mathbf{v}_{h}\right\|_{0}^{2} .
$$

Now, we multiply $(2.20)$ by $2(\nu+\sigma)$ and use the Poincaré inequality to estimate

$$
2(\nu+\sigma)\left\|\mathbf{v}_{h}\right\|_{0}^{2} \leq C\left(\nu\left|\mathbf{v}_{h}\right|_{1}^{2}+\sigma\left\|\mathbf{v}_{h}\right\|_{0}^{2}\right)
$$

Hence, we obtain

$$
\left(A+S_{h}\right)\left(\left(\mathbf{v}_{h}, q_{h}\right) ; 2(\nu+\sigma)\left(\mathbf{j}_{h} \mathbf{v}_{q_{h}}, 0\right)\right) \geq(\nu+\sigma)\left\|q_{h}\right\|_{0}^{2}-C_{1} X^{2}
$$

with a suitable constant $C_{1}$. We define for an arbitrary $\left(\mathbf{v}_{h}, q_{h}\right) \in \mathbf{V}_{h} \times Q_{h}$

$$
\left(\mathbf{w}_{h}, r_{h}\right):=\left(\mathbf{v}_{h}, q_{h}\right)+\frac{2(\nu+\sigma)}{1+C_{1}}\left(\mathbf{j}_{h} \mathbf{v}_{q_{h}}, 0\right) \in \mathbf{V}_{h} \times Q_{h}
$$

Then, we have

$$
\left(A+S_{h}\right)\left(\left(\mathbf{v}_{h}, q_{h}\right) ;\left(\mathbf{w}_{h}, r_{h}\right)\right) \geq \frac{(\nu+\sigma)}{1+C_{1}}\left\|q_{h}\right\|_{0}^{2}+\left(1-\frac{C_{1}}{1+C_{1}}\right) X^{2}=\frac{1}{1+C_{1}}\|\|\left(\mathbf{v}_{h}, q_{h}\right)\|\|^{2}
$$

and

$$
\begin{aligned}
\left\|\mid\left(\mathbf{w}_{h}, r_{h}\right)\right\| \| & \leq\|\|\left(\mathbf{v}_{h}, q_{h}\right)\left|\left\|+\frac{2(\nu+\sigma)}{1+C_{1}}\right\|\right|\left(\mathbf{j}_{h} \mathbf{v}_{q_{h}}, 0\right)\|\mid \leq\|\left(\mathbf{v}_{h}, q_{h}\right)\|\|+C(\nu+\sigma)\left\|\mathbf{j}_{h} \mathbf{v}_{q_{h}}\right\|_{1} \\
& \leq\|\|\left(\mathbf{v}_{h}, q_{h}\right)\|\|+C(\nu+\sigma)\left\|q_{h}\right\|_{0} \leq C_{2}\|\|\left(\mathbf{v}_{h}, q_{h}\right)\|\| .
\end{aligned}
$$

From (2.22) and (2.23) we conclude (2.12) with $\beta_{2}=1 /\left(C_{2}\left(1+C_{1}\right)\right)$.

Remark 2.7. Note that for $\sigma>0$ we have control over the $L^{2}$-norm of pressure and velocity uniformly with respect to $\nu>0$. However, in the case $\sigma=0$ we lose this control for $\nu \rightarrow 0$ due to the presence of the convection term $(c f .(2.17))$. If we consider the Stokes problem (i.e. $b=0$ and $\sigma=0)$, then a careful investigation shows that we still have control over the $L^{2}$-norm of the pressure with a constant independent of $\nu$ and $h$.

Remark 2.8. The unique solvability of the stabilised discrete problem (1.13) follows directly from Lemma 2.6 .

\subsection{Approximated Galerkin orthogonality}

In contrast to residual-based stabilisation schemes [11], we do not have the Galerkin orthogonality. Therefore, we investigate in this subsection the consistency error. 
Lemma 2.9. Let $(\mathbf{u}, p) \in \mathbf{V} \times Q$ be the solution of (1.3) and $\left(\mathbf{u}_{h}, p_{h}\right) \in \mathbf{V}_{h} \times Q_{h}$ be the solution of (1.13), respectively. Then,

$$
A\left(\left(\mathbf{u}-\mathbf{u}_{h}, p-p_{h}\right) ;\left(\mathbf{v}_{h}, q_{h}\right)\right)=S_{h}\left(\left(\mathbf{u}_{h}, p_{h}\right) ;\left(\mathbf{v}_{h}, q_{h}\right)\right) \quad \forall\left(\mathbf{v}_{h}, q_{h}\right) \in \mathbf{V}_{h} \times Q_{h} .
$$

Proof. We get (2.24) simply by subtracting (1.13) from (1.3).

For estimating the consistency error, we suppose that $\mathbf{b}$ is sufficiently smooth in the sense

$$
\left.\mathbf{b}\right|_{M} \in \mathbf{W}^{r, \infty}(M) \quad \forall M \in \mathcal{M}_{h}, \quad \max _{M \in \mathcal{M}_{h}}\|\mathbf{b}\|_{r, \infty, M} \leq C .
$$

Lemma 2.10. Let the fluctuation operator $\kappa_{h}$ satisfy $\mathrm{A} 2$ and $\mathbf{b}$ fulfils $(2.25)$. Then, for $(\mathbf{u}, p) \in \mathbf{H}^{r+1}(\Omega) \times$ $H^{r+1}(\Omega)$ we have

$$
\left|S_{h}\left((\mathbf{u}, p) ;\left(\mathbf{v}_{h}, q_{h}\right)\right)\right| \leq C\left(\sum_{M \in \mathcal{M}_{h}} h_{M}^{2 r}\left[\left(\tau_{M}\|\mathbf{b}\|_{r, \infty, M}^{2}+\mu_{M}\right)\|\mathbf{u}\|_{r+1, M}^{2}+\alpha_{M}\|p\|_{r+1, M}^{2}\right]\right)^{1 / 2}\left\|\left(\mathbf{v}_{h}, q_{h}\right) \mid\right\|
$$

for all $\left(\mathbf{v}_{h}, q_{h}\right) \in \mathbf{V}_{h} \times Q_{h}$.

Proof. From the definition of the stabilising term we get

$$
\left|S_{h}\left((\mathbf{u}, p) ;\left(\mathbf{v}_{h}, q_{h}\right)\right)\right| \leq\left(S_{h}((\mathbf{u}, p) ;(\mathbf{u}, p))\right)^{1 / 2}\left(S_{h}\left(\left(\mathbf{v}_{h}, q_{h}\right) ;\left(\mathbf{v}_{h}, q_{h}\right)\right)\right)^{1 / 2} \leq\left(S_{h}((\mathbf{u}, p) ;(\mathbf{u}, p))\right)^{1 / 2}\left\|\mid\left(\mathbf{v}_{h}, q_{h}\right)\right\| .
$$

Using the approximation properties of $\boldsymbol{\kappa}_{h}$, we see that

$$
S_{h}((\mathbf{u}, p) ;(\mathbf{u}, p)) \leq C \sum_{M \in \mathcal{M}_{h}} h_{M}^{2 r}\left(\tau_{M}|(\mathbf{b} \cdot \nabla) \mathbf{u}|_{r, M}^{2}+\mu_{M}|\nabla \cdot \mathbf{u}|_{r, M}^{2}+\alpha_{M}|\nabla p|_{r, M}^{2}\right)
$$

and (2.26) follows.

Remark 2.11. The assumption $\left.\mathbf{b}\right|_{M} \in \mathbf{W}^{r, \infty}(M)$ is rather restrictive in the framework of the Navier-Stokes model since $\mathbf{b}$ corresponds to a finite element function which is in general non-smooth across element borders. However, in the case $\mathcal{M}_{h}=\mathcal{T}_{h}$ the macro cells are element cells and this assumption should not be a problem. Another way to relax the smoothness assumption on $\mathbf{b}$ is the use of a modified stabilisation term, see Corollary 2.14.

\subsection{A-priori error estimate}

We get from stability and consistency an a-priori error estimate in the usual way. The important aspect is that the constant in the error bound will be independent of the viscosity $\nu$ and $h$.

Theorem 2.12. Assume A1-A3. Let $(\mathbf{u}, p) \in\left(\mathbf{H}_{0}^{1}(\Omega) \cap \mathbf{H}^{r+1}(\Omega)\right) \times\left(L_{0}^{2}(\Omega) \cap H^{r+1}(\Omega)\right)$ be the solution of (1.3) and $\left(\mathbf{u}_{h}, p_{h}\right) \in \mathbf{V}_{h} \times Q_{h}$ be the solution of the local projection method (1.13). Then, there is a positive constant $C$ independent of $\nu$ and $h$ such that

$$
\begin{aligned}
& \left\|\left|\left(\mathbf{u}-\mathbf{u}_{h}, p-p_{h}\right) \|\right| \leq C\left(\sum _ { M \in \mathcal { M } _ { h } } h _ { M } ^ { 2 r } \left[\nu+h_{M}^{2} \sigma+h_{M}^{2} \tau_{M}^{-1}+\tau_{M}\|\mathbf{b}\|_{r, \infty, M}^{2}+h_{M}^{2} \mu_{M}^{-1}+\mu_{M}\right.\right.\right. \\
& \left.\left.+h_{M}^{2} \alpha_{M}^{-1}+\alpha_{M}\right]\left(\|\mathbf{u}\|_{r+1, \Lambda(M)}^{2}+\|p\|_{r+1, \Lambda(M)}^{2}\right)\right)^{1 / 2}
\end{aligned}
$$


holds true. The choice $\tau_{M} \sim h_{M} /\|\mathbf{b}\|_{r, \infty, M}, \mu_{M} \sim h_{M}$, and $\alpha_{M} \sim h_{M}$ is asymptotically optimal and leads to

$$
\left\|\left|\left(\mathbf{u}-\mathbf{u}_{h}, p-p_{h}\right) \|\right| \leq C\left(\sum_{M \in \mathcal{M}_{h}}\left(\nu+h_{M}\right) h_{M}^{2 r}\left(\|\mathbf{u}\|_{r+1, \Lambda(M)}^{2}+\|p\|_{r+1, \Lambda(M)}^{2}\right)\right)^{1 / 2}\right.
$$

Proof. Starting with Lemma 2.6, we get an estimate for the error to the interpolants:

$$
\begin{aligned}
\left\|\left|\left(\mathbf{j}_{h} \mathbf{u}-\mathbf{u}_{h}, j_{h} p-p_{h}\right) \|\right| \leq\right. & \frac{1}{\beta_{2}} \sup _{\left(\mathbf{w}_{h}, r_{h}\right) \in \mathbf{V}_{h} \times Q_{h}} \frac{\left(A+S_{h}\right)\left(\left(\mathbf{j}_{h} \mathbf{u}-\mathbf{u}_{h}, j_{h} p-p_{h}\right) ;\left(\mathbf{w}_{h}, r_{h}\right)\right)}{\left\|\left|\left(\mathbf{w}_{h}, r_{h}\right) \|\right|\right.} \\
\leq & \frac{1}{\beta_{2}} \sup _{\left(\mathbf{w}_{h}, r_{h}\right) \in \mathbf{V}_{h} \times Q_{h}} \frac{\left(A+S_{h}\right)\left(\left(\mathbf{u}-\mathbf{u}_{h}, p-p_{h}\right) ;\left(\mathbf{w}_{h}, r_{h}\right)\right)}{\left\|\mid\left(\mathbf{w}_{h}, r_{h}\right)\right\| \|} \\
& +\frac{1}{\beta_{2}} \sup _{\left(\mathbf{w}_{h}, r_{h}\right) \in \mathbf{V}_{h} \times Q_{h}} \frac{\left(A+S_{h}\right)\left(\left(\mathbf{j}_{h} \mathbf{u}-\mathbf{u}, j_{h} p-p\right) ;\left(\mathbf{w}_{h}, r_{h}\right)\right)}{\left\|\mid\left(_{h}, r_{h}\right)\right\| \|} .
\end{aligned}
$$

Using Lemmata 2.9 and 2.10, we estimate the first term by

$$
\begin{aligned}
& \left(A+S_{h}\right)\left(\left(\mathbf{u}-\mathbf{u}_{h}, p-p_{h}\right) ;\left(\mathbf{w}_{h}, r_{h}\right)\right)=S_{h}\left((\mathbf{u}, p) ;\left(\mathbf{w}_{h}, r_{h}\right)\right) \\
& \quad \leq C\left(\sum_{M \in \mathcal{M}_{h}} h_{M}^{2 r}\left[\left(\tau_{M}\|\mathbf{b}\|_{r, \infty, M}^{2}+\mu_{M}\right)\|\mathbf{u}\|_{r+1, M}^{2}+\alpha_{M}\|p\|_{r+1, M}^{2}\right]\right)^{1 / 2}\|\|\left(\mathbf{w}_{h}, r_{h}\right)\|\|
\end{aligned}
$$

For the estimation of the second term, we consider each individual term in $\left(A+S_{h}\right)\left(\left(\mathbf{j}_{h} \mathbf{u}-\mathbf{u}, j_{h} p-p\right) ;\left(\mathbf{w}_{h}, r_{h}\right)\right)$ separately. The estimation of

$$
\nu\left(\nabla\left(\mathbf{j}_{h} \mathbf{u}-\mathbf{u}\right), \nabla \mathbf{w}_{h}\right)+\sigma\left(\mathbf{j}_{h} \mathbf{u}-\mathbf{u}, \mathbf{w}_{h}\right) \leq C\left(\sum_{M \in \mathcal{M}_{h}} h_{M}^{2 r}\left(\nu+\sigma h_{M}^{2}\right)\|\mathbf{u}\|_{r+1, \Lambda(M)}^{2}\right)^{1 / 2}\left\|\left(\mathbf{w}_{h}, r_{h}\right)\right\| \|
$$

is standard. When estimating the next three terms, we use the interpolant constructed in Theorem 2.2. Integrating by parts, we get

$$
\begin{aligned}
\left|\left((\mathbf{b} \cdot \nabla)\left(\mathbf{j}_{h} \mathbf{u}-\mathbf{u}\right), \mathbf{w}_{h}\right)\right| & =\left|\left(\mathbf{j}_{h} \mathbf{u}-\mathbf{u},(\mathbf{b} \cdot \nabla) \mathbf{w}_{h}\right)\right|=\left|\left(\mathbf{j}_{h} \mathbf{u}-\mathbf{u}, \boldsymbol{\kappa}_{h}(\mathbf{b} \cdot \nabla) \mathbf{w}_{h}\right)\right| \\
& \leq C\left(\sum_{M \in \mathcal{M}_{h}} h_{M}^{2 r+2} \tau_{M}^{-1}\|\mathbf{u}\|_{r+1, \Lambda(M)}^{2}\right)^{1 / 2}\left(S_{h}\left(\left(\mathbf{w}_{h}, 0\right) ;\left(\mathbf{w}_{h}, 0\right)\right)\right)^{1 / 2} \\
\left|\left(p-j_{h} p, \nabla \cdot \mathbf{w}_{h}\right)\right| & =\left|\left(p-j_{h} p, \kappa_{h} \nabla \cdot \mathbf{w}_{h}\right)\right| \\
& \leq C\left(\sum_{M \in \mathcal{M}_{h}} h_{M}^{2 r+2} \mu_{M}^{-1}\|p\|_{r+1, \Lambda(M)}^{2}\right)^{1 / 2}\left(S_{h}\left(\left(\mathbf{w}_{h}, 0\right) ;\left(\mathbf{w}_{h}, 0\right)\right)\right)^{1 / 2}, \\
\left|\left(r_{h}, \nabla \cdot\left(\mathbf{j}_{h} \mathbf{u}-\mathbf{u}\right)\right)\right| & =\left|\left(\nabla r_{h}, \mathbf{j}_{h} \mathbf{u}-\mathbf{u}\right)\right|=\left|\left(\boldsymbol{\kappa}_{h} \nabla r_{h}, \mathbf{j}_{h} \mathbf{u}-\mathbf{u}\right)\right| \\
& \leq C\left(\sum_{M \in \mathcal{M}_{h}} h_{M}^{2 r+2} \alpha_{M}^{-1}\|\mathbf{u}\|_{r+1, \Lambda(M)}^{2}\right)^{1 / 2}\left(S_{h}\left(\left(0, r_{h}\right) ;\left(0, r_{h}\right)\right)\right)^{1 / 2}
\end{aligned}
$$


Finally, we obtain

$$
\begin{aligned}
\mid S_{h}\left(\left(\mathbf{j}_{h} \mathbf{u}-\mathbf{u}, j_{h} p-p\right)\right. & \left.;\left(\mathbf{w}_{h}, r_{h}\right)\right) \mid \\
\leq & \left(S_{h}\left(\left(\mathbf{j}_{h} \mathbf{u}-\mathbf{u}, j_{h} p-p\right) ;\left(\mathbf{j}_{h} \mathbf{u}-\mathbf{u}, j_{h} p-p\right)\right)\right)^{1 / 2}\left(S_{h}\left(\left(\mathbf{w}_{h}, r_{h}\right) ;\left(\mathbf{w}_{h}, r_{h}\right)\right)\right)^{1 / 2} \\
\leq & C\left(\sum_{M \in \mathcal{M}_{h}} h_{M}^{2 r}\left[\left(\tau_{M}\|\mathbf{b}\|_{0, \infty, M}^{2}+\mu_{M}\right)\|\mathbf{u}\|_{r+1, \Lambda(M)}^{2}+\alpha_{M}\|p\|_{r+1, \Lambda(M)}^{2}\right]\right)^{1 / 2} \mid\left\|\left(\mathbf{w}_{h}, r_{h}\right)\right\| .
\end{aligned}
$$

Collecting all estimates above, we have shown

$$
\begin{array}{r}
\left\|\left(\mathbf{j}_{h} \mathbf{u}-\mathbf{u}_{h}, j_{h} p-p_{h}\right)\right\| \leq C\left(\sum _ { M \in \mathcal { M } _ { h } } h _ { M } ^ { 2 r } \left[\nu+h_{M}^{2} \sigma+h_{M}^{2} \tau_{M}^{-1}+\tau_{M}\|\mathbf{b}\|_{r, \infty, M}^{2}+h_{M}^{2} \mu_{M}^{-1}+\mu_{M}\right.\right. \\
\left.\left.+h_{M}^{2} \alpha_{M}^{-1}+\alpha_{M}\right]\left(\|\mathbf{u}\|_{r+1, \Lambda(M)}^{2}+\|p\|_{r+1, \Lambda(M)}^{2}\right)\right)^{1 / 2}
\end{array}
$$

By using the triangle inequality

$$
||\left(\mathbf{u}-\mathbf{u}_{h}, p-p_{h}\right)|||\leq|||\left(\mathbf{u}-\mathbf{j}_{h} \mathbf{u}, p-j_{h} p\right)|\||+||\left(\mathbf{j}_{h} \mathbf{u}-\mathbf{u}_{h}, j_{h} p-p_{h}\right)|| \mid
$$

and the approximation property

$\left\|\left(\mathbf{u}-\mathbf{j}_{h} \mathbf{u}, p-j_{h} p\right)\right\| \mid$

$$
\leq C\left(\sum_{M \in \mathcal{M}_{h}} h_{K}^{2 r}\left[\nu+h_{M}^{2} \sigma+(\nu+\sigma) h_{M}^{2}+\tau_{M}\|\mathbf{b}\|_{0, \infty, M}^{2}+\mu_{M}+\alpha_{M}\right]\left(\|\mathbf{u}\|_{r+1, \Lambda(M)}^{2}+\|p\|_{r+1, \Lambda(M)}^{2}\right)\right)^{1 / 2}
$$

we get

$$
\begin{aligned}
\left\|\left|\left(\mathbf{u}-\mathbf{u}_{h}, p-p_{h}\right) \|\right| \leq C\left(\sum_{M \in \mathcal{M}_{h}} h_{M}^{2 r}[\nu\right.\right. & +h_{M}^{2} \sigma+h_{M}^{2} \tau_{M}^{-1}+\tau_{M}\|\mathbf{b}\|_{r, \infty, M}^{2} \\
& \left.\left.+h_{M}^{2} \mu_{M}^{-1}+\mu_{M}+h_{M}^{2} \alpha_{M}^{-1}+\alpha_{M}\right]\left(\|\mathbf{u}\|_{r+1, \Lambda(M)}^{2}+\|p\|_{r+1, \Lambda(M)}^{2}\right)\right)^{1 / 2}
\end{aligned}
$$

which proves (2.27). Minimizing the upper bound results in the choice $\tau_{M} \sim h_{M} /\|\mathbf{b}\|_{r, \infty, M}, \mu_{M} \sim h_{M}$, and $\alpha_{M} \sim h_{M}$, which implies (2.28).

Remark 2.13. In comparison to the SUPG/PSPG method, we obtain with respect to the norm

$$
(v, q) \mapsto\left(\nu|v|_{1}^{2}+\sigma^{1 / 2}\|v\|_{0}^{2}+(\nu+\sigma)\|q\|_{0}^{2}\right)^{1 / 2}
$$

the same rate of convergence for equal-order interpolation [37,41]. Moreover, the SUPG/PSPG method gives additional control over

$$
\left(\sum_{K \in \mathcal{T}_{h}}\left(\tau_{K}\|(\mathbf{b} \cdot \nabla) \mathbf{v}+\nabla q\|_{0, K}^{2}+\mu_{K}\|\nabla \cdot \mathbf{v}\|_{0, K}^{2}\right)\right)^{1 / 2}
$$


Recently, it has been shown [35] that the SUPG/PSPG method also allows a separate control over the terms $\|(\mathbf{b} \cdot \nabla) \mathbf{v}\|_{0, K}$ and $\|\nabla q\|_{0, K}$ if $\sigma>0$ and the parameters $\tau_{K}$ are chosen appropriately. This behaviour of the SUPG/PSPG method is related to the behaviour of the local projection method where an additional control is only guaranteed over fluctuations of these quantities, i.e. with respect to a slightly weaker norm. Moreover, it is well-known that too much stabilisation can lead to smearing out boundary layers. Thus, the right amount of stabilisation seems to be important. Later we will see that the amount of stabilisation in the local projection method can be controlled by choosing the spaces $Y_{h}$ and $D_{h}$, respectively. This flexibility makes the local projection method very attractive.

Finally, we discuss two slightly modified approaches resulting in the same error estimates as those given in Theorem 2.12. The first modification consists in replacing the stabilising term $S_{h}$ from (1.12) by

$$
S_{h}^{1}\left(\left(\mathbf{u}_{h}, p_{h}\right) ;\left(\mathbf{v}_{h}, q_{h}\right)\right):=\sum_{M \in \mathcal{M}_{h}}\left(\tau_{M}\left(\boldsymbol{\kappa}_{h}\left(\nabla \mathbf{u}_{h}\right), \boldsymbol{\kappa}_{h}\left(\nabla \mathbf{v}_{h}\right)\right)_{M}+\alpha_{M}\left(\boldsymbol{\kappa}_{h} \nabla p_{h}, \boldsymbol{\kappa}_{h} \nabla q_{h}\right)_{M}\right)
$$

which gives control over the fluctuations of the gradients of the velocities instead of separate control over the fluctuations of the derivatives in the streamline direction and the divergence, respectively. In the second modification, we replace the stabilising term $S_{h}$ from (1.12) by a term $S_{h}^{2}$ which is spectral equivalent, i.e., there are positive constants $C_{3}, C_{4}$, independent of $\nu$ and $h$, such that

$$
C_{3} S_{h}\left(\left(\mathbf{v}_{h}, q_{h}\right) ;\left(\mathbf{v}_{h}, q_{h}\right)\right) \leq S_{h}^{2}\left(\left(\mathbf{v}_{h}, q\right) ;\left(\mathbf{v}_{h}, q_{h}\right)\right) \leq C_{4} S_{h}\left(\left(\mathbf{v}_{h}, q_{h}\right) ;\left(\mathbf{v}_{h}, q_{h}\right)\right) \quad \forall\left(\mathbf{v}_{h}, q_{h}\right) \in \mathbf{V}_{h} \times Q_{h} .
$$

Note that the choice of the parameters $\tau_{M}, \mu_{M}$, and $\alpha_{M}$ defining $S_{h}$ influences the selection of possible stabilising terms $S_{h}^{2}$ satisfying (2.34). When replacing $S_{h}$ by $S_{h}^{i}$ in (2.11), $i=1,2$, two new mesh-dependent norms appear which will be denoted by $\|\mid(\cdot, \cdot)\|_{i}, i=1,2$.

Corollary 2.14. Assume A1-A3. Let $(\mathbf{u}, p) \in\left(\mathbf{H}_{0}^{1}(\Omega) \cap \mathbf{H}^{r+1}(\Omega)\right) \times\left(L_{0}^{2}(\Omega) \cap H^{r+1}(\Omega)\right)$ be the weak solution of (1.3) and $\left(\mathbf{u}_{h}, p_{h}\right) \in \mathbf{V}_{h} \times Q_{h}$ be the solution of the local projection method (1.13) with $S_{h}$ replaced by $S_{h}^{1}$. Then, for $\sigma>0$ there is a positive constant $C$ independent of $\nu$ such that

$$
\begin{aligned}
\left\|\mid\left(\mathbf{u}-\mathbf{u}_{h}, p-p_{h}\right)\right\| \|_{1} \leq C\left(\sum_{M \in \mathcal{M}_{h}} h_{M}^{2 r}[\nu+\right. & h_{M}^{2}\left(\sigma+\sigma^{-1}|b|_{1, \infty, M}^{2}\right)+h_{M}^{2} \alpha_{M}^{-1}+\alpha_{M} \\
& \left.\left.\quad+h_{M}^{2} \tau_{M}^{-1}\left(1+\|\mathbf{b}\|_{0, \infty, M}^{2}\right)+\tau_{M}\right]\left(\|\mathbf{u}\|_{r+1, \Lambda(M)}^{2}+\|p\|_{r+1, \Lambda(M)}^{2}\right)\right)^{1 / 2}
\end{aligned}
$$

holds true. The choice $\tau_{M} \sim h_{M} \sqrt{1+\|\mathbf{b}\|_{0, \infty, M}^{2}}$ and $\alpha_{M} \sim h_{M}$ is asymptotically optimal and leads to

$$
\left\|\mid\left(\mathbf{u}-\mathbf{u}_{h}, p-p_{h}\right)\right\| \|_{1} \leq C_{\sigma}\left(\sum_{M \in \mathcal{M}_{h}}\left(\nu+h_{M}\right) h_{M}^{2 r}\left(\|\mathbf{u}\|_{r+1, \Lambda(M)}^{2}+\|p\|_{r+1, \Lambda(M)}^{2}\right)\right)^{1 / 2}
$$

with a constant $C_{\sigma}$ independent of $\nu$ but depending on $\sigma$.

Proof. A careful check shows that Lemma 2.6 with $S_{h}$ and $\||(\cdot, \cdot)|\| \mid$ replaced by $S_{h}^{1}$ and $\|\mid(\cdot, \cdot)\| \|_{1}$, respectively, is valid. Further, the additional smoothness assumption concerning $\mathbf{b}$ in Lemma 2.10 can be omitted since now the approximation properties of the fluctuation already give

$$
S_{h}^{1}((\mathbf{u}, p) ;(\mathbf{u}, p)) \leq C \sum_{M \in \mathcal{M}_{h}} h_{M}^{2 r}\left(\tau_{M}|\nabla \mathbf{u}|_{r, M}^{2}+\alpha_{M}|\nabla p|_{r, M}^{2}\right) .
$$


However, the estimates (2.29) and (2.30) in the proof of Theorem 2.12 have to be modified. Consider first (2.30):

$$
\begin{aligned}
\left|\left(p-j_{h} p, \nabla \cdot \mathbf{w}_{h}\right)\right| & =\left|\left(p-j_{h} p, \kappa_{h} \nabla \cdot \mathbf{w}_{h}\right)\right| \leq C \sum_{M \in \mathcal{M}_{h}} h_{M}^{r+1} \tau_{M}^{-1 / 2}\|p\|_{r+1, \Lambda(M)} \tau_{M}^{1 / 2}\left\|\kappa_{h} \nabla \cdot \mathbf{w}_{h}\right\|_{0, M} \\
& \leq C\left(\sum_{M \in \mathcal{M}_{h}} h_{M}^{2 r+2} \tau_{M}^{-1}\|p\|_{r+1, \Lambda(M)}^{2}\right)^{1 / 2}\left(S_{h}^{1}\left(\left(\mathbf{w}_{h}, 0\right) ;\left(\mathbf{w}_{h}, 0\right)\right)\right)^{1 / 2} .
\end{aligned}
$$

The estimation of (2.29) needs more care. We start as in the proof of Theorem 2.12

$$
\begin{aligned}
\left|\left((\mathbf{b} \cdot \nabla)\left(\mathbf{j}_{h} \mathbf{u}-\mathbf{u}\right), \mathbf{w}_{h}\right)\right| & =\left|\left(\mathbf{j}_{h} \mathbf{u}-\mathbf{u},(\mathbf{b} \cdot \nabla) \mathbf{w}_{h}\right)\right|=\left|\left(\mathbf{j}_{h} \mathbf{u}-\mathbf{u}, \boldsymbol{\kappa}_{h}(\mathbf{b} \cdot \nabla) \mathbf{w}_{h}\right)\right| \\
& \leq C \sum_{M \in \mathcal{M}_{h}} h_{M}^{r+1}\|\mathbf{u}\|_{r+1, \Lambda(M)}\left\|\boldsymbol{\kappa}_{h}(\mathbf{b} \cdot \nabla) \mathbf{w}_{h}\right\|_{0, M} .
\end{aligned}
$$

Let $\overline{\mathbf{b}}$ be the $L^{2}$-projection of $\mathbf{b}$ in the space of piecewise constant functions with respect to the macro decomposition $\mathcal{M}_{h}$. Using the $L^{2}$-stability of $\boldsymbol{\kappa}_{h}$, an inverse inequality, and $\boldsymbol{\kappa}_{h}(\overline{\mathbf{b}} \cdot \nabla) \mathbf{w}_{h}=\overline{\mathbf{b}} \cdot \boldsymbol{\kappa}_{h}\left(\nabla \mathbf{w}_{h}\right)$, we get

$$
\begin{aligned}
\left\|\boldsymbol{\kappa}_{h}(\mathbf{b} \cdot \nabla) \mathbf{w}_{h}\right\|_{0, M} & \leq\left\|\boldsymbol{\kappa}_{h}((\mathbf{b}-\overline{\mathbf{b}}) \cdot \nabla) \mathbf{w}_{h}\right\|_{0, M}+\left\|\boldsymbol{\kappa}_{h}(\overline{\mathbf{b}} \cdot \nabla) \mathbf{w}_{h}\right\|_{0, M} \\
& \leq C h_{M}|\mathbf{b}|_{1, \infty, M}\left\|\nabla \mathbf{w}_{h}\right\|_{0, M}+\|\mathbf{b}\|_{0, \infty, M}\left\|\boldsymbol{\kappa}_{h}\left(\nabla \mathbf{w}_{h}\right)\right\|_{0, M} \\
& \leq C|\mathbf{b}|_{1, \infty, M}\left\|\mathbf{w}_{h}\right\|_{0, M}+\|\mathbf{b}\|_{0, \infty, M}\left\|\boldsymbol{\kappa}_{h}\left(\nabla \mathbf{w}_{h}\right)\right\|_{0, M} .
\end{aligned}
$$

Since $\sigma>0$, we end up with

$$
\begin{aligned}
& \left|\left((\mathbf{b} \cdot \nabla)\left(\mathbf{j}_{h} \mathbf{u}-\mathbf{u}\right), \mathbf{w}_{h}\right)\right| \leq C \sum_{M \in \mathcal{M}_{h}} h_{M}^{r+1}\|\mathbf{u}\|_{r+1, \Lambda(M)}\left(|\mathbf{b}|_{1, \infty, M}\left\|\mathbf{w}_{h}\right\|_{0, M}+\|\mathbf{b}\|_{0, \infty, M}\left\|\boldsymbol{\kappa}_{h}\left(\nabla \mathbf{w}_{h}\right)\right\|_{0, M}\right) \\
& \quad \leq C\left(\sum_{M \in \mathcal{M}_{h}} h_{M}^{2 r}\left[h_{M}^{2} \sigma^{-1}|\mathbf{b}|_{1, \infty, M}^{2}+h_{M}^{2} \tau_{M}^{-1}\|\mathbf{b}\|_{0, \infty, M}^{2}\right]\|\mathbf{u}\|_{r+1, \Lambda(M)}^{2}\right)^{1 / 2}\left(\sigma\left\|\mathbf{w}_{h}\right\|_{0}^{2}+S_{h}^{1}\left(\left(\mathbf{w}_{h}, 0\right) ;\left(\mathbf{w}_{h}, 0\right)\right)\right)^{1 / 2}
\end{aligned}
$$

The remaining terms can be estimated as in the proof of Theorem 2.12. Finally, we obtain

$$
\begin{aligned}
\left\|\left|\left(\mathbf{u}-\mathbf{u}_{h}, p-p_{h}\right)\right|\right\|_{1} \leq C\left(\sum_{M \in \mathcal{M}_{h}} h_{M}^{2 r}[\nu+\right. & h_{M}^{2}\left(\sigma+\sigma^{-1}|\mathbf{b}|_{1, \infty, M}^{2}\right)+h_{M}^{2} \alpha_{M}^{-1}+\alpha_{M} \\
& \left.\left.+h_{M}^{2} \tau_{M}^{-1}\left(1+\|\mathbf{b}\|_{0, \infty, M}^{2}\right)+\tau_{M}\right]\left(\|\mathbf{u}\|_{r+1, \Lambda(M)}^{2}+\|p\|_{r+1, \Lambda(M)}^{2}\right)\right)^{1 / 2}
\end{aligned}
$$

which is the first statement of the corollary. Minimizing the upper bound gives $\tau_{M} \sim h_{M} \sqrt{1+\|\mathbf{b}\|_{0, \infty, M}^{2}}$ and $\alpha_{M} \sim h_{M}$, which implies (2.35).

We come back to the second modification which replaces $S_{h}$ by a spectrally equivalent stabilisation term $S_{h}^{2}$. We assume (2.34), the consistency estimate

$$
\left|S_{h}^{2}\left((\mathbf{u}, p) ;\left(\mathbf{v}_{h}, q_{h}\right)\right)\right| \leq C h^{r+1 / 2}\left(\|\mathbf{u}\|_{r+1}+\|p\|_{r+1}\right)\|\|\left(\mathbf{v}_{h}, q_{h}\right)\|\| \quad \forall\left(\mathbf{v}_{h}, q_{h}\right) \in \mathbf{V}_{h} \times Q_{h}
$$


and the approximation property

$$
\left|S_{h}^{2}\left(\left(\mathbf{j}_{h} \mathbf{u}-\mathbf{u}, j_{h} p-p\right) ;\left(\mathbf{v}_{h}, q_{h}\right)\right)\right| \leq C h^{r+1 / 2}\left(\|\mathbf{u}\|_{r+1}+\|p\|_{r+1}\right)\|\|\left(\mathbf{v}_{h}, q_{h}\right) \mid \| \quad \forall\left(\mathbf{v}_{h}, q_{h}\right) \in \mathbf{V}_{h} \times Q_{h}
$$

to be satisfied.

Corollary 2.15. Assume A1, A3, and $\tau_{M}, \mu_{M}, \alpha_{M} \sim h_{M}$. Let $(\mathbf{u}, p) \in\left(\mathbf{H}_{0}^{1}(\Omega) \cap \mathbf{H}^{r+1}(\Omega)\right) \times\left(L_{0}^{2}(\Omega) \cap H^{r+1}(\Omega)\right)$ be the solution of (1.3) and $\left(\mathbf{u}_{h}, p_{h}\right) \in \mathbf{V}_{h} \times Q_{h}$ be the solution of the local projection method (1.13) with $S_{h}$ replaced by $S_{h}^{2}$ satisfying (2.34), (2.36), and (2.37). Then, there is a positive constant $C$ independent of $\nu$ and $h$ such that

$$
\left\|\left(\mathbf{u}-\mathbf{u}_{h}, p-p_{h}\right)\right\|_{2} \leq C\left(\nu^{1 / 2}+h^{1 / 2}\right) h^{r}\left(\|\mathbf{u}\|_{r+1}+\|p\|_{r+1}\right)
$$

holds true.

Proof. A careful check shows that Lemma 2.6 with $S_{h}$ and $\|\mid(\cdot, \cdot)\| \|$ replaced by $S_{h}^{2}$ and $\left.\||(\cdot, \cdot)|\|\right|_{2}$, respectively, is valid. Lemma 2.10 is replaced by (2.36). Now following the lines of proof of Theorem 2.12 and bounding $S_{h}$ by $C_{3}^{-1} S_{h}^{2}$, we get

$$
\left\|\left(\mathbf{j}_{h} \mathbf{u}-\mathbf{u}_{h}, j_{h} p-p_{h}\right)\right\|_{2} \leq C\left(\nu^{1 / 2}+h^{1 / 2}\right) h^{r}\left(\|\mathbf{u}\|_{r+1}+\|p\|_{r+1}\right) .
$$

The statement follows from the triangle inequality.

\section{SCHEMES BASED ON LOCAL PROJECTION ONTO COARSER MESHES}

The triangulation $\mathcal{T}_{h}$ consists of generic cells $K$ whereas the macro mesh $\mathcal{M}_{h}$ consists of macro cells $M$. The partition $\mathcal{T}_{h}$ is formed by a suitable refinement of the macro mesh $\mathcal{M}_{h}$ which will be indicated by the notation $\mathcal{M}_{h}=\mathcal{T}_{2 h}$.

\subsection{Simplices}

Let $\widehat{M}$ be the unit $d$-simplex with the vertices $\hat{a}_{i}, i=1, \ldots, d+1$, and the barycenter $\hat{a}_{0}$. The refinement of $\widehat{M}$ is done in the following way. Each child $\widehat{K}_{i}$ is given by the vertices $\hat{a}_{0}$ and $\hat{a}_{j}, j \neq i$, see left picture in Figure 1 for the $2 \mathrm{~d}$ case. Let $F_{M}: \widehat{M} \rightarrow M$ denote the affine mapping from the reference macro $\widehat{M}$ onto the macro cell $M \in \mathcal{M}_{h}$. This mapping defines cells $K \in \mathcal{T}_{h}$ by setting $K=F_{M}\left(\widehat{K}_{i}\right), i=1, \ldots, d+1, M \in \mathcal{M}_{h}$, see right picture in Figure 1 for the $2 \mathrm{~d}$ case. For a function $v: M \rightarrow \mathbb{R}$, we define $\hat{v}:=v \circ F_{M}: \widehat{M} \rightarrow \mathbb{R}$. Furthermore, we consider the affine mapping $F_{K}: \widehat{K} \rightarrow K$ from a reference $d$-simplex $\widehat{K}$ onto an arbitrary cell $K \in \mathcal{T}_{h}$.

We choose for the approximation of velocity and pressure the finite element space of continuous, piecewise polynomials of degree $r \in \mathbb{N}$. Let the projection space consist of discontinuous, piecewise polynomials of degree $r-1$ on $\mathcal{T}_{2 h}$, i.e. shortly

$$
Y_{h} / D_{h}=P_{r, h} / P_{r-1,2 h}^{\text {disc }}
$$

where

$$
\begin{aligned}
P_{r, h} & :=\left\{v \in H^{1}(\Omega):\left.v\right|_{K} \circ F_{K} \in P_{r}(\widehat{K}) \quad \forall K \in \mathcal{T}_{h}\right\} \\
P_{r-1,2 h}^{\text {disc }} & :=\left\{v \in L^{2}(\Omega):\left.v\right|_{M} \circ F_{M} \in P_{r-1}(\widehat{M}) \quad \forall M \in \mathcal{T}_{2 h}\right\} .
\end{aligned}
$$

We define the auxiliary space $\widehat{Y}(\widehat{M})$ as a counterpart of $Y_{h}(M)$ by

$$
\widehat{Y}(\widehat{M}):=\left\{w \in H_{0}^{1}(\widehat{M}):\left.w\right|_{\widehat{K}_{i}} \in P_{r}\left(\widehat{K}_{i}\right), \quad i=1, \ldots, d+1\right\},
$$



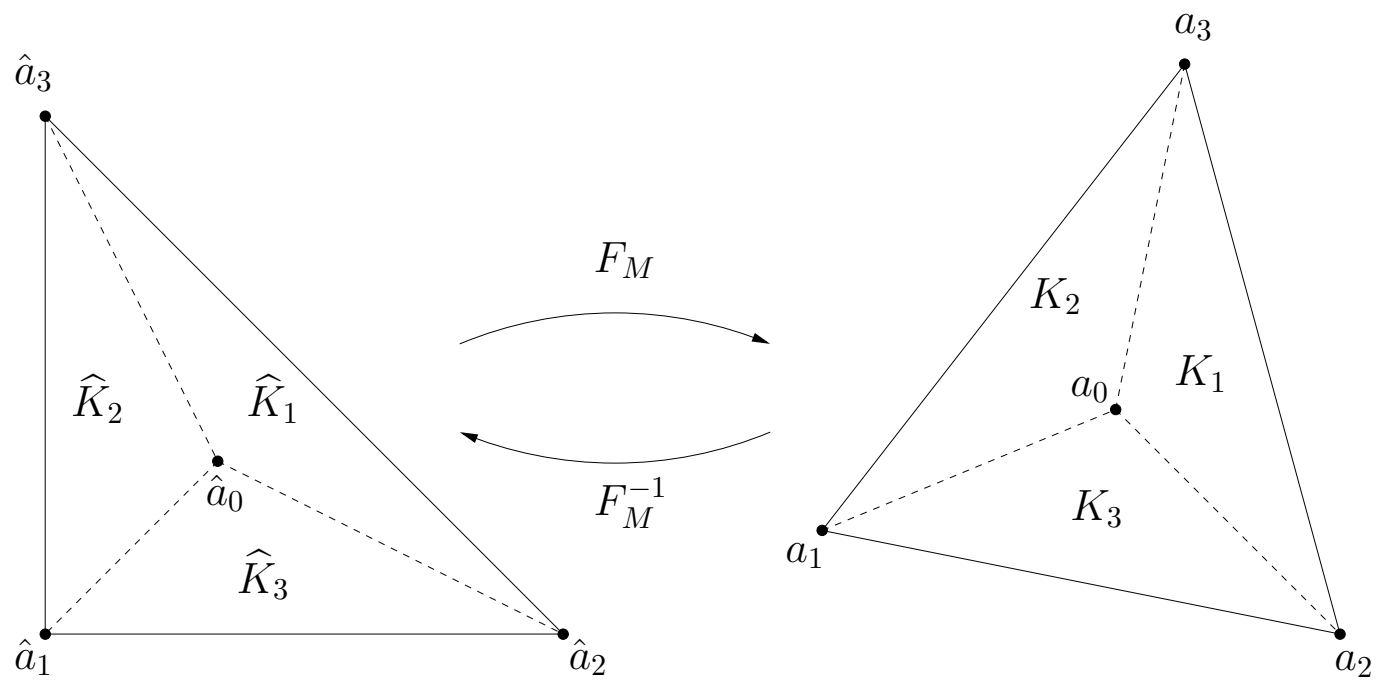

Figure 1. Reference macro triangle (left) and macro triangle $M \in \mathcal{M}_{h}$ (right).

i.e., on the reference $d$-simplex $\widehat{M}$ we have

$$
\widehat{Y}(\widehat{M})=\left\{\hat{v}: \hat{v} \circ F_{M}^{-1} \in Y_{h}(M)\right\}
$$

where $\widehat{K}_{i}$ are children of $\widehat{M}$ resulting from the decomposition of $\widehat{M}$. The existence of the interpolation operators satisfying the Assumption A1 is obvious. Further, we can choose the local projection $\pi_{M}$ in such a way that $\pi_{M}=i d$ on the subspace $P_{r-1}$. This guarantees Assumption A2 too.

Lemma 3.1. Let the local projection scheme be defined for the pair $Y_{h} / D_{h}=P_{r, h} / P_{r-1,2 h}^{\text {disc }}$ with an arbitrary but fixed polynomial degree $r \in \mathbb{N}$. Then, on shape regular simplicial meshes the local inf-sup condition A3 holds with a constant $\beta_{1}$ independent of $h$.

Proof. We make use of the reference transformation $F_{M}: \widehat{M} \rightarrow M$ and observe that the local inf-sup condition $\mathrm{A} 3$ can be in virtue of the constant $\left|\operatorname{det} D F_{M}\right|$ investigated merely on the reference macro

$$
\inf _{q \in D_{h}(M)} \sup _{v \in Y_{h}(M)} \frac{(q, v)_{M}}{\|q\|_{0, M}\|v\|_{0, M}}=\inf _{\hat{q} \in P_{r-1}(\widehat{M})} \sup _{\hat{v} \in \widehat{Y}(\widehat{M})} \frac{(\hat{q}, \hat{v})_{\widehat{M}}}{\|\hat{q}\|_{0, \widehat{M}}\|\hat{v}\|_{0, \widehat{M}}} .
$$

Let $\hat{q} \in P_{r-1}(\widehat{M})$ be arbitrarily chosen. By $\hat{b}: \widehat{M} \rightarrow \mathbb{R}$ we denote the piecewise linear hat function associated with $\hat{a}_{0}$, i.e., $\left.\hat{b}\right|_{\widehat{K}_{i}}$ is linear, $\hat{b}\left(\hat{a}_{0}\right)=1, \hat{b}\left(\hat{a}_{i}\right)=0, i=1, \ldots, d+1$, and we set $\hat{v}:=\hat{q} \cdot \hat{b}$. We note that $\hat{v} \in \widehat{Y}(\widehat{M})$ since $\hat{v}$ is continuous, $\left.\hat{v}\right|_{\partial \widehat{M}}=0$, and $\left.\hat{v}\right|_{\widehat{K}_{i}} \in P_{r}\left(\widehat{K}_{i}\right)$ on each child $\widehat{K}_{i}, i=1, \ldots, d+1$. Since $\hat{b}>0$ in $\widehat{M}$, we state that

$$
\hat{q} \mapsto(\hat{q}, \hat{q} \cdot \hat{b})_{0, \widehat{M}}^{1 / 2}
$$

is a norm on the space $P_{r-1}(\widehat{M})$. Using the fact that all norms on finite dimensional spaces are equivalent, we get

$$
(\hat{q}, \hat{v})_{\widehat{M}}=(\hat{q}, \hat{q} \cdot \hat{b})_{\widehat{M}} \geq \beta_{1}\|\hat{q}\|_{0, \widehat{M}}^{2}
$$

where the constant $\beta_{1}$ is clearly independent of $h$. Due to $|\hat{b}(\hat{x})| \leq 1 \forall \hat{x} \in \widehat{M}$, we have on the other hand

$$
\|\hat{v}\|_{0, \widehat{M}} \leq\|\hat{q}\|_{0, \widehat{M}}
$$




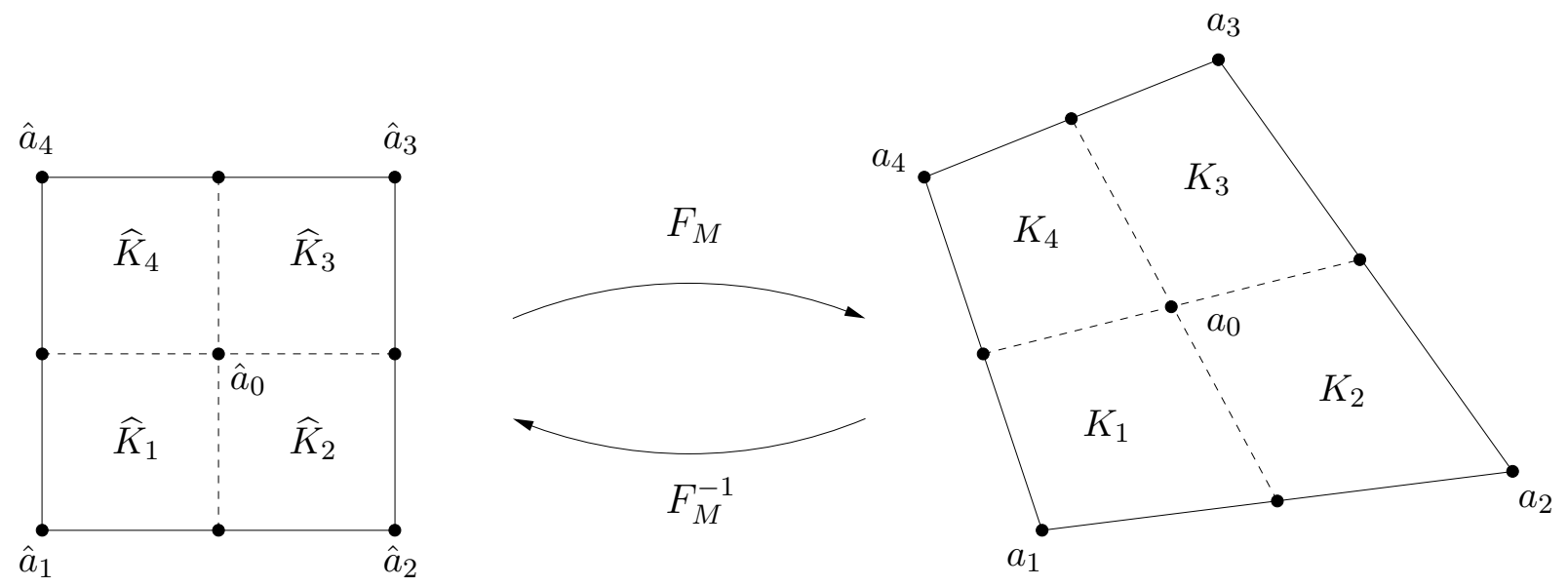

Figure 2. Reference macro quadrilateral (left) and macro quadrilateral $M \in \mathcal{M}_{h}$ (right).

From (3.1)-(3.3) we conclude A3.

\subsection{Quadrilaterals and hexahedra}

Let $\widehat{M}=(-1,1)^{d}$ be the reference hyper-cube with the vertices $\hat{a}_{i}, i=1, \ldots, 2^{d}$, and the barycenter $\hat{a}_{0} . \widehat{M}$ is refined into $2^{d}$ congruent cubes $\widehat{K}_{i}, i=1, \ldots, 2^{d}$. Let $F_{M}: \widehat{M} \rightarrow M$ be the multilinear reference mapping. The refinement of $\widehat{M}$ induces a refinement of $M$ into $2^{d}$ cells, see Figure 2 for the $2 \mathrm{~d}$ case. The union of all these cells forms the principal mesh

$$
\mathcal{T}_{h}=\bigcup_{M \in \mathcal{M}_{h}}\left\{F_{M}\left(\widehat{K}_{i}\right): i=1, \ldots, 2^{d}\right\} .
$$

Furthermore, we consider the multilinear mapping $F_{K}: \widehat{K} \rightarrow K$ from the reference hyper-cube $\widehat{K}=(-1,1)^{d}$ onto an arbitrary cell $K \in \mathcal{T}_{h}$.

One can define the projection space $D_{h}$ in two ways, namely as an image of a space on the reference macro $\widehat{M}$ or directly on the macro $M$. This leads to different finite element spaces, so we distinguish between both variants of the projection space $D_{h}$. The mapped version of $D_{h}$ takes advantage of fixing the projection space locally on the reference macro. However, the interpolation property A2 turns out to be missing on arbitrary families of meshes [3,34]. To keep the notation clear, we use the extra superscript ' $m$ ' for mapped finite element spaces $D_{h}$.

\subsubsection{Projection spaces based on mapped finite elements}

First, we consider the following finite element pair

$$
Y_{h} / D_{h}=Q_{r, h} / Q_{r-1,2 h}^{\text {disc,m }}
$$

where

$$
\begin{aligned}
Q_{r, h} & :=\left\{v \in H^{1}(\Omega):\left.v\right|_{K} \circ F_{K} \in Q_{r}(\widehat{K}) \quad \forall K \in \mathcal{T}_{h}\right\}, \\
Q_{r-1,2 h}^{\mathrm{disc}, \mathrm{m}} & :=\left\{v \in L^{2}(\Omega):\left.v\right|_{M} \circ F_{M} \in Q_{r-1}(\widehat{M}) \quad \forall M \in \mathcal{T}_{2 h}\right\} .
\end{aligned}
$$

The auxiliary space $\widehat{Y}(\widehat{M})$ is defined in analogy to the simplicial macros, i.e., on the reference hyper-cube we have

$$
\widehat{Y}(\widehat{M})=\left\{w \in H_{0}^{1}(\widehat{M}):\left.w\right|_{\widehat{K}_{i}} \in Q_{r}\left(\widehat{K}_{i}\right), \quad i=1, \ldots, 2^{d}\right\}
$$


where $\widehat{K}_{i}$ are children of $\widehat{M}$ resulting from the decomposition of $\widehat{M}$. The construction of interpolation operators satisfying the Assumption A1 is standard. Moreover, since $P_{r-1}(M) \subset Q_{r-1}^{\mathrm{disc}, \mathrm{m}}(M)$ holds true, the Bramble-Hilbert lemma provides the optimal interpolation properties A2 on families of shape regular meshes.

Following the lines of proof of Lemma 3.1 for establishing Assumption A3, we find that in general $\left|\operatorname{det} D_{F} M\right|$ is no longer constant, and consequently (3.1) holds only in special cases (parallelograms/parallelepipeds). Before we continue our investigation of the Assumption A3, we recall some general properties of the multilinear reference mapping $F_{T}: \widehat{T} \rightarrow T$ from $\widehat{T}=(-1,1)^{d}$ onto an arbitrary quadrilaterals/hexahedra $T . F_{T}$ can be expanded as follows

$$
F_{T}(\hat{x})=m_{T}+B_{T} \hat{x}+G_{T}(\hat{x})
$$

where $m_{T}:=F_{T}(0), B_{T}:=D F_{T}(0)$, and $G_{T}(\hat{x})=F_{T}(\hat{x})-F_{T}(0)-D F_{T}(0)(\hat{x})$. Furthermore, we define by means of the Euclidian norm the distortion parameter

$$
\gamma_{T}:=\sup _{\hat{x} \in \widehat{T}}\left\|B_{T}^{-1} D G_{T}(\hat{x})\right\|
$$

and assume $\gamma_{T} \leq \gamma<1$. Then, the mapping $F_{T}$ is one-to-one and from [36], Lemma 2 , the estimate

$$
C^{-1} d !\left(1-\gamma_{T}\right)^{d} h_{T}^{d} \leq\left|\operatorname{det} D F_{T}(\hat{x})\right| \leq C d !\left(1+\gamma_{T}\right)^{d} h_{T}^{d}
$$

follows. Moreover, on a family of uniformly refined meshes we have

$$
\lim _{h \rightarrow 0} \gamma_{T}=0 .
$$

For a parallelogram/parallelepiped $T$, the reference mapping $F_{T}$ is affine and $\gamma_{T}=0$.

Lemma 3.2. Let the local projection scheme be defined for the pair $Y_{h} / D_{h}=Q_{r, h} / Q_{r-1,2 h}^{\text {disc,m }}$ with an arbitrary but fixed polynomial degree $r \in \mathbb{N}$. Then, the local inf-sup condition A3 holds with a constant $\beta_{1}$ independent of $h$.

Proof. From (3.5) we get

$$
\|q\|_{0, M}^{2} \leq C d !\left(1+\gamma_{M}\right)^{d} h_{M}^{d}\|\hat{q}\|_{0, \widehat{M}}^{2} \quad \forall q \in D_{h}(M) .
$$

Let $\hat{b}: \widehat{M} \rightarrow \mathbb{R}$ be the piecewise multilinear hat function associated with $\hat{a}_{0}$, i.e., $\hat{b}_{\widehat{K}_{i}} \in Q_{1}\left(\widehat{K}_{i}\right), i=1, \ldots, 2^{d}$, $\hat{b}\left(\hat{a}_{0}\right)=1, \hat{b}\left(\hat{a}_{i}\right)=0, i=1, \ldots, 2^{d}$. For an arbitrary $q \in D_{h}(M)$ we choose $v(x):=(\hat{q} \cdot \hat{b}) \circ F_{M}^{-1}(x)$ where $\hat{q} \in Q_{r-1}(\widehat{M})$. Since $\hat{q} \cdot \hat{b}$ is continuous on the closure of $\widehat{M},\left.(\hat{q} \cdot \hat{b})\right|_{\widehat{K}_{i}} \in Q_{r}\left(\widehat{K}_{i}\right), i=1, \ldots, 2^{d}$, and $\left.\hat{b}\right|_{\partial \widehat{M}}=0$, we have $\hat{v}(\hat{x}):=\hat{q}(\hat{x}) \hat{b}(\hat{x}) \in \widehat{Y}(\widehat{M})$. Then, it follows from the estimate $(3.5)$

$$
\begin{aligned}
(q, v)_{M} & =\int_{M} q(x) v(x) \mathrm{d} x=\int_{\widehat{M}} \hat{q}(\hat{x}) \hat{v}(\hat{x})\left|\operatorname{det} D F_{M}(\hat{x})\right| \mathrm{d} \hat{x}=\int_{\widehat{M}} \hat{q}(\hat{x}) \hat{q}(\hat{x}) \hat{b}(\hat{x})\left|\operatorname{det} D F_{M}(\hat{x})\right| \mathrm{d} \hat{x} \\
& \geq C d !\left(1-\gamma_{M}\right)^{d} h_{M}^{d} \int_{\widehat{M}}(\hat{q}(\hat{x}))^{2} \hat{b}(\hat{x}) \mathrm{d} \hat{x} .
\end{aligned}
$$

The equivalence of norms on the finite dimensional space $Q_{r-1}(\widehat{M})$ implies

$$
\|\hat{q} \cdot \sqrt{\hat{b}}\|_{0, \widehat{M}} \geq C\|\hat{q}\|_{0, \widehat{M}} \quad \forall \hat{q} \in Q_{r-1}(\widehat{M})
$$

and hence

$$
(q, v)_{K} \geq C d !\left(1-\gamma_{M}\right)^{d} h_{M}^{d}\|\hat{q}\|_{0, \widehat{M}}^{2} .
$$


Using $|\hat{b}(\hat{x})| \leq 1 \forall \hat{x} \in \widehat{M}$, we get

$$
\|v\|_{0, M}^{2} \leq \int_{\widehat{M}}(\hat{q}(\hat{x}))^{2}\left|\operatorname{det} D F_{M}(\hat{x})\right| \mathrm{d} \hat{x} \leq C d !\left(1+\gamma_{M}\right)^{d} h_{M}^{d}\|\hat{q}\|_{0, \widehat{M}}^{2} .
$$

From (3.6)-(3.8) it follows immediately

$$
\forall q \in D_{h}(M) \quad \exists v \in Y_{h}(M): \quad \frac{(q, v)_{M}}{\|q\|_{0, M}\|v\|_{0, M}} \geq C\left(\frac{1-\gamma_{M}}{1+\gamma_{M}}\right)^{d} \geq C\left(\frac{1-\gamma}{1+\gamma}\right)^{d}=: \beta_{1}
$$

and thus, the local inf-sup condition A3 is proven.

Alternatively, one can choose a smaller projection spaces $D_{h}$ as follows

$$
P_{r-1,2 h}^{\text {disc } m}:=\left\{v \in L^{2}(\Omega):\left.v\right|_{M} \circ F_{M} \in P_{r-1}(\widehat{M}) \quad \forall M \in \mathcal{T}_{2 h}\right\} .
$$

This results in more stabilisation in the sense that the stabilising term vanishes on the smaller subset $P_{r-1,2 h}^{\text {disc } m} \subset$ $Q_{r-1,2 h}^{\text {disc }, m}$. The Assumption A1 holds without any change. However to guarantee Assumption A2 we have to restrict ourselves to suitably refined families of meshes, see [3] for quadrilaterals and [34] for hexahedra.

Corollary 3.3. Let the local projection scheme be defined for the pair $Y_{h} / D_{h}=Q_{r, h} / P_{r-1,2 h}^{\text {disc,m }}$ with an arbitrary but fixed polynomial degree $r \in \mathbb{N}$. Then, the local inf-sup condition A3 holds with a constant $\beta_{1}$ independent of $h$.

Remark 3.4. To discuss other choices of the projection space $D_{h}$, we mention that we have in the case $Y_{h} / D_{h}=Q_{r, h} / Q_{r-1,2 h}^{\text {disc } m}$ the relation

$$
\operatorname{dim} Y_{h}(M)=(2 r-1)^{d} \geq r^{d}=\operatorname{dim} D_{h}(M)
$$

in particular, for $r=1$ the dimensions of both spaces coincide. In the case $r \geq 2$, one could think of choosing a larger projection space in order to minimise the stabilisation. A possible candidate would be $D_{h}=Q_{r, 2 h}^{\text {disc,m }}$ since now

$$
\operatorname{dim} Y_{h}(M)=(2 r-1)^{d} \geq(r+1)^{d}=\operatorname{dim} D_{h}(M), \quad r \geq 2 .
$$

The Assumption A1 holds without any change, A2 would be satisfied with a higher rate than required. However, whether the inf-sup condition A3 holds, is an open problem.

3.2.2. Projection spaces based on unmapped finite elements

We choose for the projection space $D_{h}$ the space of discontinuous, piecewise polynomials of degree $r-1$ posed directly on the generic cells, i.e.

where

$$
Y_{h} / D_{h}=Q_{r, h} / P_{r-1,2 h}^{\text {disc }}
$$

$$
\begin{aligned}
Q_{r, h} & :=\left\{v \in H^{1}(\Omega):\left.v\right|_{K} \circ F_{K} \in Q_{r}(\widehat{K}) \quad \forall K \in \mathcal{T}_{h}\right\}, \\
P_{r-1,2 h}^{\text {disc }} & :=\left\{v \in L^{2}(\Omega):\left.v\right|_{M} \in P_{r-1}(M) \quad \forall M \in \mathcal{T}_{2 h}\right\} .
\end{aligned}
$$

Again, the Assumption A1 holds without any change. In contrast to the mapped version the approximation property A2 is now fulfilled for any shape regular family of meshes. The local inf-sup condition follows from

Lemma 3.5. Let the local projection scheme be defined for the pair $Y_{h} / D_{h}=Q_{r, h} / P_{r-1,2 h}^{\text {disc }}$ with an arbitrary but fixed polynomial degree $r \in \mathbb{N}$. Then, on quadrilateral/hexahedral meshes the local inf-sup condition A3 holds with a constant $\beta_{1}$ independent of $h$.

Proof. Since $P_{r-1,2 h}^{\text {disc }}$ is contained in $Q_{r-1,2 h}^{\text {disc,m }}$, the proof is a straightforward consequence of Lemma 3.2. 


\section{SCHEMES BASED on ENRICHMENT OF APPROXimation SPACES}

This class of schemes takes advantage of constructing the finite element spaces $Y_{h}$ and $D_{h}$ on the same mesh. There are some indications of using only one principal mesh given recently in [11]. However, there the choice of polynomial spaces of order at least $r \geq 2$ and the construction of a nodal fluctuation operator is not optimal with respect to the order of convergence. We propose a novel method based on the enrichment of approximation spaces in order to satisfy the local inf-sup condition A3. The main benefit of this enrichment is the reduced stencil of the stiffness matrix and the avoidance of the difficult handling of data structures in the local projection methods which use coarser meshes. We present the method for two types of mesh geometries.

\subsection{Simplices}

Let

$$
\hat{b}(\hat{x}):=(d+1)^{d+1} \prod_{i=1}^{d+1} \hat{\lambda}_{i}(\hat{x})
$$

be the bubble function which takes the value 1 in the barycentre of the reference simplex $\widehat{K}_{\text {. Thereby }} \hat{\lambda}_{i}$, $i=1, \ldots, d+1$, are barycentric coordinates on $\widehat{K}$. Furthermore, we define the enriched space

$$
P_{r}^{\text {bubble }}(\widehat{K}):=P_{r}(\widehat{K})+\hat{b} \cdot P_{r-1}(\widehat{K}) .
$$

Let

$$
Y_{h} / D_{h}:=P_{r, h}^{\text {bubble }} / P_{r-1, h}^{\text {disc }}
$$

be the pair of finite element spaces defined via the reference mapping

$$
\begin{aligned}
P_{r, h}^{\text {bubble }}: & =\left\{v \in H^{1}(\Omega):\left.v\right|_{K} \circ F_{K} \in P_{r}^{\text {bubble }}(\widehat{K}) \quad \forall K \in \mathcal{T}_{h}\right\}, \\
P_{r-1, h}^{\text {disc }}: & :=\left\{v \in L^{2}(\Omega):\left.v\right|_{K} \circ F_{K} \in P_{r-1}(\widehat{K}) \quad \forall K \in \mathcal{T}_{h}\right\} .
\end{aligned}
$$

Obviously, the Assumptions A1 and A2 are fulfilled. At a first glance, the enriched space seems to be large, but a more careful look shows

$$
P_{r}(\widehat{K})+\hat{b} \cdot P_{r-1}(\widehat{K})=P_{r}(\widehat{K}) \oplus\left(\hat{b} \cdot \sum_{i=1}^{d} \tilde{P}_{r-i}(\widehat{K})\right)
$$

where $\tilde{P}_{r}(\widehat{K})=\operatorname{span}\left(\left\{\prod_{i=1}^{d} \hat{x}_{i}^{\alpha_{i}}, \quad \sum_{i=1}^{d} \alpha_{i}=r, \quad\left(\hat{x}_{1}, \ldots, \hat{x}_{d}\right) \in \widehat{K}\right\}\right)$ is a monomial space. Using the fact that the bubble part of the space $P_{r}(\widehat{K})$ is $\hat{b} \cdot P_{r-(d+1)}(\widehat{K})$, we have

$$
\begin{aligned}
\operatorname{dim} \widehat{Y}(\widehat{K}) & =\left(\begin{array}{c}
r-(d+1)+d \\
d
\end{array}\right)+\sum_{i=1}^{d}\left[\left(\begin{array}{c}
r-i+d \\
d
\end{array}\right)-\left(\begin{array}{c}
r-i+d-1 \\
d
\end{array}\right)\right] \\
& =\left(\begin{array}{c}
r-1 \\
d
\end{array}\right)+\left(\begin{array}{c}
r-1+d \\
d
\end{array}\right)-\left(\begin{array}{c}
r-1 \\
d
\end{array}\right)=\operatorname{dim} P_{r-1}(\widehat{K}) .
\end{aligned}
$$

The chosen enrichment is minimal with respect to the required inequality (2.2).

Lemma 4.1. Let the local projection scheme be defined for the pair $Y_{h} / D_{h}=P_{r, h}^{\text {bubble }} / P_{r-1, h}^{\text {disc }}$ with an arbitrary but fixed polynomial degree $r \in \mathbb{N}$. Then, the local inf-sup condition A3 holds with a constant $\beta_{1}$ independent of $h$. 
Proof. Since the reference mapping is affine, the proof of the local inf-sup stability can be performed using the identity (3.1) for the reference cell $\widehat{K}$. For an arbitrary $\hat{q} \in P_{r-1}(\widehat{K})$ we choose $\hat{v}:=\hat{b} \cdot \hat{q}$ with $\hat{b}$ defined by (4.1). Since $\hat{b} \cdot \hat{q} \in P_{r}^{\text {bubble }}(\widehat{K})$ and $\left.(\hat{b} \cdot \hat{q})\right|_{\partial \widehat{K}}=0$, we have $\hat{v} \in \widehat{Y}(\widehat{K})$, and with the analogous argumentation as in the proof of the Lemma 3.1, we get the required positive lower bound $\beta_{1}$ independent of the mesh size $h$.

\subsection{Quadrilaterals and hexahedra}

As for the method based on projection onto coarser meshes, we will distinguish between mapped

$$
P_{r-1, h}^{\mathrm{disc}, \mathrm{m}}:=\left\{v \in L^{2}(\Omega):\left.v\right|_{K} \circ F_{K} \in P_{r-1}(\widehat{K}) \quad \forall K \in \mathcal{T}_{h}\right\}
$$

and unmapped

$$
P_{r-1, h}^{\text {disc }}:=\left\{v \in L^{2}(\Omega):\left.v\right|_{K} \in P_{r-1}(K) \quad \forall K \in \mathcal{T}_{h}\right\}
$$

finite element spaces for the projection space $D_{h}$. In order to obtain the optimal order of the interpolation error for the mapped projection space, families of uniformly refined quadrilateral/hexahedral meshes are required, see again [3,34], whereas for the unmapped spaces the interpolation property A2 holds on general shape regular meshes. We extend the approximation spaces in order to ensure the local inf-sup condition A3.

\subsubsection{Projection spaces based on mapped finite elements}

Let

$$
\hat{b}(\hat{x})=\prod_{i=1}^{d}\left(1-\hat{x}_{i}^{2}\right) \in Q_{2}(\widehat{K}), \quad \hat{x}=\left(\hat{x}_{1}, \ldots, \hat{x}_{d}\right) \in \widehat{K}, \quad d=2,3,
$$

be a bubble function associated with the reference cell $\widehat{K}=(-1,1)^{d}$. The enriched finite element space is set to be

$$
Q_{r}^{\text {bubble }, 1}(\widehat{K}):=Q_{r}(\widehat{K}) \oplus \operatorname{span}\left(\hat{b} \hat{x}_{i}^{r-1}, i=1, \ldots, d\right) .
$$

We define a pair of finite element spaces

$$
Y_{h} / D_{h}:=Q_{r, h}^{\text {bubble, } 1} / P_{r-1, h}^{\text {disc,m }}
$$

via the reference mapping

$$
Q_{r, h}^{\text {bubble }, 1}:=\left\{v \in H^{1}(\Omega):\left.v\right|_{K} \circ F_{K} \in Q_{r}^{\text {bubble, }, 1}(\widehat{K}) \quad \forall K \in \mathcal{T}_{h}\right\} .
$$

We note that in general the functions of spaces $Q_{r, h}^{\text {bubble, } 1}, P_{r-1, h}^{\text {disc,m }}$ are not polynomials. Since $Q_{r}(\widehat{K}) \subset$ $Q_{r}^{\text {bubble, } 1}(\widehat{K})$, the Assumption A1 is satisfied. Assumption A2 holds on uniformly refined meshes, see [3,34].

Lemma 4.2. Let the local projection scheme be defined for the pair $Y_{h} / D_{h}=Q_{r, h}^{\text {bubble, } 1} / P_{r-1, h}^{\text {disc,m }}$ with an arbitrary but fixed polynomial degree $r \in \mathbb{N}$. Then, the local inf-sup condition A3 holds with a constant $\beta_{1}$ independent of $h$.

Proof. For an arbitrary $q \in D_{h}(K)$ we choose $v(x):=(\hat{q} \cdot \hat{b}) \circ F_{K}^{-1}(x)$ where $\hat{b} \geq 0$ is the bubble function from (4.2), $\hat{q} \in P_{r-1}(\widehat{K})$. Since $\hat{q}=\hat{q}_{0}+\hat{q}_{1}$ with $\hat{q}_{0} \in \operatorname{span}\left(x_{i}^{r-1}, i=1, \ldots, d\right)$ and $\hat{q}_{1} \in Q_{r-2}$, we have $\hat{v}(\hat{x}):=\hat{q}(\hat{x}) \hat{b}(\hat{x}) \in Q_{r}^{\text {talbubble, } 1}(\widehat{K})$. Then, we proceed as in the proof of Lemma 3.2 and get from (3.5)

$$
\forall q \in D_{h}(K) \quad \exists v \in Y_{h}(K): \quad \frac{(q, v)_{K}}{\|q\|_{0, K}\|v\|_{0, K}} \geq C\left(\frac{1-\gamma_{K}}{1+\gamma_{K}}\right)^{d} \geq C\left(\frac{1-\gamma}{1+\gamma}\right)^{d}=: \beta_{1} .
$$

This implies the local inf-sup condition A3. 
TABLE 1. Numbers of non-zero matrix entries in the two-dimensional case for the two-level $\left(Q_{r, h} / Q_{r-1,2 h}^{\text {disc,m }}\right)$ and for the enrichment approach $\left(Q_{r, h}^{\text {bubble,1 }} / P_{r-1}^{\text {disc,m }}\right)$, respectively.

\begin{tabular}{llll}
\hline \multicolumn{3}{c}{ Two-level approach } \\
\hline \hline Object & \# object & dofs per object & Matrix entries per dof \\
\hline Macro vertex & $\mathcal{O}\left(N^{2} / 4\right)$ & 1 & $(4 r+1)^{2}$ \\
Macro edge & $\mathcal{O}\left(N^{2} / 2\right)$ & $2 r-1$ & $(4 r+1)(2 r+1)$ \\
Macro cell & $\mathcal{O}\left(N^{2} / 4\right)$ & $(2 r-1)^{2}$ & $(2 r+1)^{2}$ \\
\hline \multicolumn{4}{c}{ Enrichment approach } \\
\hline \hline Object & $\#$ object & dofs per object & Matrix entries per dof \\
\hline Vertex & $\mathcal{O}\left(N^{2}\right)$ & 1 & $(2 r+1)^{2}+4 \cdot 2$ \\
Edge & $\mathcal{O}\left(2 N^{2}\right)$ & $r-1$ & $(2 r+1)(r+1)+2 \cdot 2$ \\
Cell & $\mathcal{O}\left(N^{2}\right)$ & $(r-1)^{2}+2$ & $(r+1)^{2}+2$ \\
\hline
\end{tabular}

TABLE 2. Numbers of non-zero matrix entries in the three-dimensional case for the two-level $\left(Q_{r, h} / Q_{r-1,2 h}^{\text {disc,m }}\right)$ and for the enrichment approach $\left(Q_{r, h}^{\text {bubble,1 }} / P_{r-1}^{\text {disc,m }}\right)$, respectively.

\begin{tabular}{llll}
\hline \multicolumn{4}{c}{ Two-level approach } \\
\hline \hline Object & $\#$ object & dofs per object & Matrix entries per dof \\
\hline Macro vertex & $\mathcal{O}\left(N^{3} / 8\right)$ & 1 & $(4 r+1)^{3}$ \\
Macro edge & $\mathcal{O}\left(3 N^{3} / 8\right)$ & $2 r-1$ & $(4 r+1)^{2}(2 r+1)$ \\
Macro face & $\mathcal{O}\left(3 N^{3} / 8\right)$ & $(2 r-1)^{2}$ & $(4 r+1)(2 r+1)^{2}$ \\
Macro cell & $\mathcal{O}\left(N^{3} / 8\right)$ & $(2 r-1)^{3}$ & $(2 r+1)^{3}$ \\
\hline \multicolumn{4}{c}{ Enrichment approach } \\
\hline \hline Object & $\#$ object & dofs per object & Matrix entries per dof \\
\hline Vertex & $\mathcal{O}\left(N^{3}\right)$ & 1 & $(2 r+1)^{3}+8 \cdot 3$ \\
Edge & $\mathcal{O}\left(3 N^{3}\right)$ & $r-1$ & $(2 r+1)^{2}(r+1)+4 \cdot 3$ \\
Face & $\mathcal{O}\left(3 N^{3}\right)$ & $(r-1)^{2}$ & $(2 r+1)(r+1)^{2}+2 \cdot 3$ \\
Cell & $\mathcal{O}\left(N^{3}\right)$ & $(r-1)^{3}+3$ & $(r+1)^{3}+3$ \\
\hline
\end{tabular}

Remark 4.3. A comparison of the dimensions of the spaces $Y_{h}(M)$ and $D_{h}(M)$ shows that

$$
\operatorname{dim} \widehat{Y}(\widehat{K})=(r-1)^{d}+d \geq\left(\begin{array}{c}
r-1+d \\
d
\end{array}\right)=\operatorname{dim} P_{r-1}(\widehat{K}) \quad \forall r \in \mathbb{N} \forall d \in \mathbb{N}
$$

In particular, the enrichment is optimal for biquadratic and bicubic elements on quadrilaterals and for triquadratic elements on hexahedra.

Remark 4.4. Note that the space $Q_{r}^{\text {bubble, }, 1}(\widehat{K})$ has for $r \geq 2$ exactly $d$ basis functions more than $Q_{r}(\widehat{K})$, independent of $r$.

Remark 4.5. To get an impression on the efficiency of the new enrichment approach, we consider the matrixblock corresponding to one scalar component and compare asymptotically the numbers of non-zero entries for a decomposition of $\Omega=(0,1)^{d}$ into squares/cubes of edge size $1 / N$, see Tables 1 and 2 . Since the inner degrees of freedom (dofs) dominate for high order elements $(r \gg 1)$, we have asymptotically $\mathcal{O}\left(4 N^{2} r^{4}\right)$ and $\mathcal{O}\left(8 N^{3} r^{6}\right)$ nonzero entries for the two-level approach whereas the enrichment technique produces only $\mathcal{O}\left(N^{2} r^{4}\right)$ and $\mathcal{O}\left(N^{3} r^{6}\right)$ non-zero entries. This effect is less distinct for moderate $r$. For example, in the case $r=2$ and $d=2$, we get asymptotically $\mathcal{O}\left(144 N^{2}\right)$ compared to $\mathcal{O}\left(75 N^{2}\right)$ non-zero entries. 


\subsubsection{Projection spaces based on unmapped finite elements}

In order to satisfy A2 on general meshes, we propose an alternative way for setting the finite element pair $Y_{h} / D_{h}$. We choose the space

$$
Q_{r}^{\text {bubble }, 2}(\widehat{K}):=Q_{r}(\widehat{K})+\hat{b} \cdot Q_{r-1}(\widehat{K})
$$

with the bubble function $\hat{b}$ from (4.2) and define the enriched space

$$
Q_{r, h}^{\text {bubble }, 2}:=\left\{v \in H^{1}(\Omega):\left.v\right|_{K} \circ F_{K} \in Q_{r}^{\text {bubble }, 2}(\widehat{K}) \quad \forall K \in \mathcal{T}_{h}\right\}
$$

Now, our alternative choice is

$$
Y_{h} / D_{h}=Q_{r, h}^{\text {bubble }, 2} / P_{r-1, h}^{\text {disc }} .
$$

Then, the properties A1 and A2 are naturally fulfilled.

Lemma 4.6. Let the local projection scheme be defined for the pair $Y_{h} / D_{h}=Q_{r, h}^{\text {bubble }, 2} / P_{r-1, h}^{\text {disc }}$ with an arbitrary but fixed polynomial degree $r \in \mathbb{N}$. Then, the local inf-sup condition A3 holds with a constant $\beta_{1}$ independent of $h$.

Proof. For an arbitrary $q \in D_{h}$, we set $v=q \cdot b_{K}$ where $b_{K}(x):=\left(\hat{b} \circ F_{K}^{-1}\right)(x)$ with the bubble function $\hat{b}$ defined by (4.2) and the reference mapping $F_{K}$. Since $q \in P_{r-1}(K)$, we find $\hat{q} \in Q_{r-1}(\widehat{K})$. Consequently, $\hat{b} \cdot \hat{q} \in Q_{r}^{\text {bubble, } 2}(\widehat{K})$ and $\left.(\hat{b} \cdot \hat{q})\right|_{\partial \widehat{K}}=0$. Then, we have $\hat{v}:=\hat{b} \cdot \hat{q} \in \widehat{Y}(\widehat{K})$ and $v \in Y_{h}(K)$. In analogy to the proof of Lemma 4.2 , we obtain from (3.5) with $T=K$, the norm equivalence

$$
\|\hat{q} \cdot \sqrt{\hat{b}}\|_{0, \widehat{K}} \geq C\|\hat{q}\|_{0, \widehat{K}} \quad \forall \hat{q} \in Q_{r-1}(\widehat{K}),
$$

and $\|\hat{v}\|_{0, \widehat{K}} \leq\|\hat{q}\|_{0, \widehat{K}}$, the following

$$
\forall q \in D_{h}(K) \quad \exists v \in Y_{h}(K): \quad \frac{(q, v)_{K}}{\|q\|_{0, K}\|v\|_{0, K}} \geq C\left(\frac{1-\gamma_{K}}{1+\gamma_{K}}\right)^{d} \geq C\left(\frac{1-\gamma}{1+\gamma}\right)^{d}=: \beta_{1}
$$

which is the statement of the lemma.

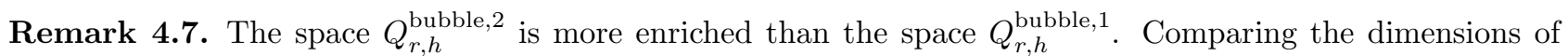
spaces $Y_{h}(K)$ and $D_{h}(K)$, we can guess that the enriched space could be reduced. However, the question of the validity of the local inf-sup condition is still an open problem.

\section{Relation to SUbGRid MODELling}

The idea of subgrid modelling goes back to Guermond and has firstly been applied to a scalar transport equation [19]. It is based on a scale separation of the underlying finite element space

$$
Y_{h}=Y_{H} \oplus Y_{h}^{H}
$$

where $Y_{H}$ stands for the space of large scales and $Y_{h}^{H}$ for the space of small scales. Associated with the scale separation is a suitable projection operator $P_{H}: Y_{h} \rightarrow Y_{H} \subset Y_{h}$ which is the identity on the subspace $Y_{H}$. Let $\bar{\kappa}_{h}:=i d-P_{H}$ denote the fluctuation operator. We assume that the finite element space $Y_{H}$ is based on a shape regular decomposition of the domain into cells $M \in \mathcal{M}_{h}$ of diameter $h_{M}$. Then, a stabilising term of the form

$$
S\left(u_{h}, v_{h}\right)=\sum_{M \in \mathcal{M}_{h}} h_{M}\left(\nabla \bar{\kappa}_{h} u_{h}, \nabla \bar{\kappa}_{h} v_{h}\right)_{M} \quad \text { or } \quad S\left(u_{h}, v_{h}\right)=\sum_{M \in \mathcal{M}_{h}} h_{M}\left((b \cdot \nabla) \bar{\kappa}_{h} u_{h},(b \cdot \nabla) \bar{\kappa}_{h} v_{h}\right)_{M}
$$

has been proposed to add to the standard Galerkin approach [15,19]. These stabilisation terms can be interpreted as an artificial diffusion term or an artificial diffusion in the streamline direction for the subscales which are 
represented by $Y_{h}^{H}$. This technique has been developed in different directions, for an extension to time-dependent convection-diffusion problems see e.g. [30]. Scale separation plays also an important role in large eddy simulation of turbulent flows, see [28].

The scale separation can be realised in different ways. In the two-level approach, $Y_{h}$ and $Y_{H}$ are standard finite element spaces on different refinement levels (we indicate this by writing $Y_{H}=Y_{2 h}$ ) and $Y_{h}^{H}$ consists just of those hierarchical basis functions which are missing in the coarse space $Y_{2 h}$ to generate $Y_{h}$. Another view is to consider $Y_{h}$ as a finite element space $Y_{H}$ enriched by $Y_{h}^{H}$ which contains suitable functions, e.g. higher order polynomials. However, both variants differ from the local projection approach since the stabilisation term in the subgrid modelling approach is based on gradients of fluctuations $\nabla\left(i d-P_{H}\right) u_{h}$ whereas the local projection method uses fluctuations of the gradients $\left(i d-\pi_{h}\right) \nabla u_{h}$.

In applications, the projection $P_{H}: Y_{h} \rightarrow Y_{2 h}$ in the two-level approach has been often chosen as the global Lagrange interpolant $I_{2 h, r}$ into $Y_{2 h}[4,7-9,11,32]$. This leads to a stabilising term of the form

$$
\mathcal{S}_{h}^{3}\left(\left(\mathbf{u}_{h}, p_{h}\right) ;\left(\mathbf{v}_{h}, q_{h}\right)\right)=\sum_{M \in \mathcal{M}_{h}} \tau_{M}\left(\nabla \overline{\boldsymbol{\kappa}}_{h} \mathbf{u}_{h}, \nabla \overline{\boldsymbol{\kappa}}_{h} \mathbf{v}_{h}\right)_{M}+\alpha_{M}\left(\nabla \bar{\kappa}_{h} p_{h}, \nabla \bar{\kappa}_{h} q_{h}\right)_{M}
$$

instead of $S_{h}$ given by (1.12). In the following subsections, we study the relations between the stabilising terms $S_{h}^{1}$ given by $(2.33)$ and $S_{h}^{3}$.

\subsection{Two-level approach on piecewise linear elements}

We consider first the case where $\mathcal{T}_{h}$ is generated from a refinement of a shape regular triangulation $\mathcal{T}_{2 h}$ by connecting the barycentre of each macro cell with its vertices, see Figure 1 for the $2 \mathrm{~d}$ case. Let $Y_{h}$ and $Y_{2 h}$ denote the spaces of continuous, piecewise linear finite elements associated with the triangulations $\mathcal{T}_{h}$ and $\mathcal{T}_{2 h}$, respectively.

Lemma 5.1. Let $d \geq 1, \pi_{2 h, 0}$ be the $L^{2}$-projection onto the space $P_{0,2 h}^{\text {disc }}$ of piecewise constant functions, and $I_{2 h, 1}: Y_{h} \rightarrow Y_{2 h}$ be the Lagrange interpolant into the space $P_{1,2 h}$ of continuous, piecewise linear functions. Then, we have

$$
\left.\pi_{2 h, 0}\left(\nabla v_{h}\right)\right|_{M}=\nabla I_{2 h, 1}\left(\left.v_{h}\right|_{M}\right) \quad \forall v_{h} \in P_{1, h}, \forall M \in \mathcal{T}_{2 h}
$$

Thus, the discrete problems of the local projection method and the subgrid modelling approach coincide.

Proof. We consider the case of an arbitrary dimension $d \geq 1$. We restrict ourselves to the scalar case since the assertion for the vector-valued case follows immediately from the scalar one by a component-wise application.

Let $\left.v_{h}\right|_{M}$ be the restriction of a finite element function $v_{h} \in Y_{h}$ onto a macro simplex $M \in \mathcal{T}_{2 h}$. Furthermore, let $\lambda_{i}, i \stackrel{M}{=} 1, \ldots, d+1$, denote the barycentric coordinates with respect to the simplex $M$. Defining the continuous, piecewise linear function

$$
\varphi_{0}(x)=(d+1) \lambda_{i}(x), \quad x \in K_{i}, i=1, \ldots, d+1
$$

we can represent $\left.v_{h}\right|_{M}$ by its nodal functionals $N_{i}(v)=v\left(a_{i}\right), i=0, \ldots, d+1$, as

$$
\left.v_{h}\right|_{M}=\sum_{i=1}^{d+1} N_{i}\left(v_{h}\right) \lambda_{i}+\tilde{N}_{0}\left(v_{h}\right) \varphi_{0}
$$

with

$$
\tilde{N}_{0}(v)=N_{0}(v)-\frac{1}{d+1} \sum_{i=1}^{d+1} N_{i}(v) .
$$


Since $N_{i}\left(\varphi_{0}\right)=0, i=1, \ldots, d+1$, we have

$$
I_{2 h, 1} v_{h}=\sum_{i=1}^{d+1} N_{i}\left(v_{h}\right) \lambda_{i}
$$

from which

$$
\nabla I_{2 h, 1} v_{h}=\sum_{i=1}^{d+1} N_{i}\left(v_{h}\right) \nabla \lambda_{i}
$$

follows. Let $\nabla_{h}$ denote the piecewise applied gradient operator. Taking into consideration that $\nabla_{h} v_{h}$ is constant on each subdomain $K_{j}, j=1, \ldots, d+1$, and that $\left|K_{j}\right|=|K| /(d+1)$, we compute the $L^{2}$-projection onto $P_{0}(M)$ to be

$$
\pi_{2 h, 0}\left(\nabla_{h} v_{h}\right)=\left.\frac{1}{d+1} \sum_{j=1}^{d+1} \nabla_{h} v_{h}\right|_{K_{j}} .
$$

Next we get from $(5.2)$ for $j=1, \ldots, d+1$

$$
\begin{gathered}
\left.\nabla v_{h}\right|_{K_{j}}=\sum_{i=1}^{d+1} N_{i}\left(v_{h}\right) \nabla \lambda_{i}+(d+1) \tilde{N}_{0}\left(v_{h}\right) \nabla \lambda_{j} \\
\left.\frac{1}{d+1} \sum_{j=1}^{d+1} \nabla v_{h}\right|_{K_{j}}=\sum_{i=1}^{d+1} N_{i}\left(v_{h}\right) \nabla \lambda_{i}+\tilde{N}_{0}\left(v_{h}\right) \nabla \sum_{j=1}^{d+1} \lambda_{j}=\sum_{i=1}^{d+1} N_{i}\left(v_{h}\right) \nabla \lambda_{i} .
\end{gathered}
$$

Since $\left.\pi_{2 h, 0}\left(\nabla v_{h}\right)\right|_{M}=\nabla I_{2 h, 1}\left(\left.v_{h}\right|_{M}\right)$ holds true, the stabilising terms in both approaches are identical.

Remark 5.2. In general we have that

$$
\left.\pi_{2 h, r-1} \nabla_{h} v_{h}\right|_{M} \neq \nabla I_{2 h, r} v_{h} \quad \forall v_{h} \in P_{r, h}, r \geq 2
$$

where $\pi_{2 h, r-1}$ is the $L^{2}$-projection onto the space $P_{r-1,2 h}^{\text {disc }}$ of discontinuous, piecewise polynomials of degree $r-1$ on the coarse mesh $\mathcal{T}_{2 h}$ and $I_{2 h, r}: Y_{h} \rightarrow P_{r, 2 h}$ is the Lagrange interpolant into the space of continuous, piecewise polynomials of degree $r$ on the coarse mesh $\mathcal{T}_{2 h}$. Similarly, we have that in general on quadrilateral or hexahedral meshes $\mathcal{T}_{2 h}$

$$
\left.\pi_{2 h, r-1} \nabla_{h} v_{h}\right|_{M} \neq \nabla I_{2 h, r} v_{h} \quad \forall v_{h} \in Q_{r, h}^{d}, d \geq 2, r \geq 1
$$

where $\pi_{2 h, r-1}$ is the $L^{2}$-projection onto the space $Q_{r-1,2 h}^{\text {disc }}$ of discontinuous, piecewise polynomials of degree $r-1$ in each variable and $I_{2 h, r}: Y_{h} \rightarrow Q_{r, 2 h}$ is the Lagrange interpolant into the space of continuous, piecewise polynomials of degree $r$ in each variable. As an example, we consider the case $r=2, d=1$. We get for the reference macro $\widehat{M}=(-1,+1)$ and the piecewise quadratic function

$$
\widehat{v}(\hat{x})= \begin{cases}4 \hat{x}(1-\hat{x}) & \text { if } 0 \leq \hat{x} \leq 1 \\ 0 & \text { if }-1 \leq \hat{x}<0\end{cases}
$$

the relation

$$
\hat{\pi}_{2 h, 1} \hat{\nabla} \hat{v}=-\hat{x} \neq 0=\hat{\nabla} \hat{I}_{2 h, 2} \hat{v} .
$$

Thus, in general the two approaches, subgrid modelling and local projection, do not lead to the same stabilisation term. However, we will see later that this does not automatically exclude the possibility of spectral equivalence of the stabilisation terms. 


\subsection{Enriched piecewise linear elements}

In the previous section we have seen that both methods, the local projection scheme and the subgrid modelling, results for the two-level approach with $Y_{h}=P_{1, h}$ and $D_{h}=P_{0,2 h}^{\text {disc }}$ in the same stabilisation term. Now we will show that the same is also true for enriched piecewise linear elements, i.e., $Y_{h}=P_{1, h}^{\text {bubble }}$ and $D_{h}=P_{0, h}^{\text {disc }}$.

Lemma 5.3. Let $d \geq 1, \pi_{h, 0}$ be the $L^{2}$-projection onto the space $P_{0, h}^{\mathrm{disc}}$ of piecewise constant functions, and $I_{h, 1}: Y_{h} \rightarrow P_{1, h}$ be the Lagrange interpolant into the space $P_{1, h}$ of continuous, piecewise linear functions. Then, we have

$$
\left.\pi_{h, 0}\left(\nabla v_{h}\right)\right|_{K}=\nabla I_{h, 1}\left(\left.v_{h}\right|_{K}\right) \quad \forall v_{h} \in P_{1, h}^{\text {bubble }}, \forall K \in \mathcal{T}_{h}
$$

Thus, the discrete problems of the local projection method and the subgrid modelling approach coincide.

Proof. For simplicity of notation we present the proof for the scalar case. The extension to the vector-valued case in the space $Y_{h}^{d}$ is straightforward. We consider a simplex $K \in \mathcal{T}_{h}$ with the vertices $a_{i}, i=1, \ldots, d+1$, the barycentre $a_{0}$, and the barycentric coordinates $\lambda_{i}, i=1, \ldots, d+1$. The restriction $\left.v_{h}\right|_{K}$ of a finite element function $v_{h} \in Y_{h}$ onto $K$ can be represented by its nodal functionals $N_{i}(v)=v\left(a_{i}\right), i=0, \ldots, d+1$, as

$$
\left.v_{h}\right|_{K}=\sum_{i=1}^{d+1} N_{i}\left(v_{h}\right) \lambda_{i}+\tilde{N}_{0}\left(v_{h}\right) b=I_{h, 1} v_{h}+\widetilde{N}_{0}\left(v_{h}\right) b
$$

where

$$
\tilde{N}_{0}(v)=N_{0}(v)-\frac{1}{d+1} \sum_{i=1}^{d+1} N_{i}(v) \quad \text { and } \quad b=(d+1)^{d+1} \prod_{i=1}^{d+1} \lambda_{i}
$$

is the scaled product of all barycentric coordinates $\lambda_{i}, i=1, \ldots, d+1$. Applying the gradient gives

$$
\left.\nabla v_{h}\right|_{K}=\nabla\left(I_{h, 1} v_{h}\right)+N_{0}\left(v_{h}\right) \nabla b
$$

Since $\nabla\left(I_{h, 1} v_{h}\right)$ is constant on $K$, we have $\pi_{h, 0} \nabla\left(I_{h, 1} v_{h}\right)=\nabla\left(I_{h, 1} v_{h}\right)$. Hence, it remains to show

$$
\pi_{h, 0}(\nabla b)=\frac{1}{|K|} \int_{K} \nabla b \mathrm{dx}=\mathbf{0}
$$

This follows immediately from the Gaussian theorem since $b$ vanishes on $\partial K$. Consequently, $\left.\pi_{h, 0}\left(\nabla v_{h}\right)\right|_{K}=$ $\nabla I_{h, 1}\left(\left.v_{h}\right|_{K}\right)$ holds and the stabilising terms in both approaches are identical.

\subsection{Spectral equivalence of the stabilising terms on simplices}

Now, we will show on simplices the spectral equivalence between the stabilising terms $S_{h}^{3}$ given by (5.1) and $S_{h}^{1}$ given by (2.33). To this end, it is sufficient to show the existence of positive constants $C_{3}, C_{4}$ such that

$$
C_{3}\left\|\kappa_{h} \nabla w_{h}\right\|_{0, M} \leq\left\|\nabla \bar{\kappa}_{h} w_{h}\right\|_{0, M} \leq C_{4}\left\|\kappa_{h} \nabla w_{h}\right\|_{0, M} \quad \forall w_{h} \in Y_{h}, \quad \forall M \in \mathcal{M}_{h}
$$

First we consider the two-level approach.

Lemma 5.4. Let $Y_{h}=P_{r, h}, D_{h}=P_{r-1,2 h}^{\text {disc }}, \pi_{2 h, r-1}$ be the $L^{2}$-projection onto $D_{h}, \kappa_{h}=i d-\pi_{2 h, r-1}, I_{2 h, r}$ be the Lagrange interpolant in $P_{r, 2 h}$, and $\bar{\kappa}_{h}=i d-I_{2 h, r}$. Then, the stabilising terms $S_{h}^{3}$ and $S_{h}^{1}$ are spectrally equivalent. 
Proof. For $M \in \mathcal{T}_{2 h}$ let $F_{M}: \widehat{M} \rightarrow M$ be the affine mapping from the reference macro cell $\widehat{M}$ onto the cell $M$ and $B_{M}=D F_{M}$. The $L^{2}$-projection $\pi_{2 h, r-1}$ and the Lagrange interpolant $I_{2 h, r}$ are invariant with respect to affine transformations, i.e., denoting the corresponding operators on the reference cell by $\hat{\pi}$ and $\hat{I}$, we have

$$
\left(\widehat{\pi_{2 h, r-1}} w\right)=\hat{\pi} \hat{w}, \quad \widehat{I_{2 h, r} w}=\hat{I} \hat{w}
$$

and the corresponding relations for the fluctuation operators

$$
\widehat{\kappa_{h} \nabla w}=\hat{\kappa} \widehat{\nabla w}, \quad \widehat{\kappa_{h} w}=\widehat{\bar{\kappa}} \widehat{\nabla w} .
$$

Now using the transformation formulas $\widehat{\nabla v}=B_{M}^{-T} \hat{\nabla} \hat{v}$ and $\hat{\nabla} \hat{v}=B_{M}^{T} \widehat{\nabla v}$ [13], Chapter 3.1, we obtain

$$
\begin{gathered}
\left\|\kappa_{h} \nabla w\right\|_{0, M}=\left|\operatorname{det} B_{M}\right|^{1 / 2}\left\|\widehat{\kappa_{h} \nabla w}\right\|_{0, \widehat{M}}=\left|\operatorname{det} B_{M}\right|^{1 / 2}\left\|\hat{\kappa} B_{M}^{-T} \hat{\nabla} \hat{w}\right\|_{0, \widehat{M}} \leq\left|\operatorname{det} B_{M}\right|^{1 / 2}\left\|B_{M}^{-1}\right\|\|\hat{\kappa} \hat{\nabla} \hat{w}\|_{0, \widehat{M}} \\
\|\hat{\nabla} \hat{\bar{\kappa}} \hat{w}\|_{0, \widehat{M}}=\left\|\hat{\nabla} \widehat{\overline{\kappa_{h}} w}\right\|_{0, \widehat{M}}=\left|\operatorname{det} B_{M}\right|^{-1 / 2}\left\|B_{M}^{T} \nabla \bar{\kappa}_{h} w\right\|_{0, M} \leq\left|\operatorname{det} B_{M}\right|^{-1 / 2}\left\|B_{M}\right\|\left\|\nabla \bar{\kappa}_{h} w\right\|_{0, M}
\end{gathered}
$$

where $\left\|B_{M}\right\|$ and $\left\|B_{M}^{-1}\right\|$ are the matrix norms of $B_{M}$ and $B_{M}^{-1}$ which are induced by the Euclidean vector norm.

If there is a constant $C$ such that $\|\hat{\kappa} \hat{\nabla} \hat{w}\|_{0, \widehat{M}} \leq C\|\hat{\nabla} \hat{\bar{\kappa}} \hat{w}\|_{0, \widehat{M}}$, we get from (5.4), (5.5), and $\left\|B_{M}^{-1}\right\|\left\|B_{M}\right\| \leq C$ which holds for shape regular meshes,

$$
\left\|\kappa_{h} \nabla w\right\|_{0, M} \leq C_{3}^{-1}\left\|\nabla \bar{\kappa}_{h} w\right\|_{0, M}
$$

which is the left hand side of (5.3). The proof of the right hand side follows from $\|\hat{\nabla} \hat{\kappa} \hat{w}\|_{0, \widehat{M}} \leq C\|\hat{\kappa} \hat{\nabla} \hat{w}\|_{0, \widehat{M}}$ by the same arguments.

To show the missing inequalities on the reference element, we consider the mappings

$$
\hat{w} \mapsto\|\hat{\kappa} \hat{\nabla} \hat{w}\|_{0, \widehat{M}}, \quad \hat{w} \mapsto\|\hat{\nabla} \hat{\bar{\kappa}} \hat{w}\|_{0, \widehat{M}}
$$

which are norms on the finite dimensional factor spaces

$$
P_{r}(\widehat{M}) /\{\widehat{w}: \hat{\kappa} \hat{\nabla} \hat{w}=\mathbf{0}\} \quad \text { and } \quad P_{r}(\widehat{M}) /\{\hat{w}: \hat{\nabla} \hat{\kappa} \hat{w}=\mathbf{0}\}
$$

respectively. Let us assume that $\hat{\kappa} \hat{\nabla} \hat{w}=\mathbf{0}$. Then, we conclude

$$
\hat{\nabla} \hat{w}=\hat{\pi} \hat{\nabla} \hat{w} \in\left(P_{r-1}(\widehat{M})\right)^{d} \quad \Longrightarrow \quad \hat{w} \in P_{r}(\widehat{M}) \quad \Longrightarrow \quad \hat{I} \hat{w}=\hat{w} \quad \Longrightarrow \quad \hat{\nabla} \hat{\bar{\kappa}} \hat{w}=\mathbf{0} .
$$

Conversely, assuming $\hat{\nabla} \hat{\bar{\kappa}} \hat{w}=\mathbf{0}$, and taking into consideration that $\hat{w}$ is continuous on $\widehat{M}$, we obtain

$$
\hat{w}=\hat{I} \hat{w}+\text { const } \in P_{r}(\widehat{M}) \quad \Longrightarrow \quad \hat{\nabla} \hat{w}=\hat{\nabla} \hat{I} \hat{w} \in\left(P_{r-1}(\widehat{M})\right)^{d} \quad \Longrightarrow \quad \hat{\pi} \hat{\nabla} \hat{w}=\hat{\nabla} \hat{w} . \quad \Longrightarrow \quad \hat{\kappa} \hat{\nabla} \hat{w}=\mathbf{0} .
$$

Thus, the two factor spaces coincide and the missing inequalities follow from the equivalence of norms on finite dimensional spaces.

Let us turn to the case of enriched finite element spaces $Y_{h}$.

Lemma 5.5. Let $Y_{h}=P_{r, h}^{\text {bubble }}$ defined in Section 4.1, $D_{h}=P_{r-1, h}^{\text {disc }}, \pi_{h, r-1}$ be the $L^{2}$-projection onto $D_{h}, I_{h, r}$ be the Lagrange interpolant in $P_{r, h}, \kappa_{h}=i d-\pi_{h, r-1}$, and $\bar{\kappa}_{h}=i d-I_{h, r}$. Then, the stabilising terms $S_{h}^{3}$ and $S_{h}^{1}$ are spectrally equivalent. 
Proof. First, by using the affine transformation $F_{K}: \widehat{K} \rightarrow K$ from the reference cell $\widehat{K}$ onto $K$, we can show, as in the proof of Lemma 5.4, that it suffices to establish the corresponding estimations on the reference cell. As before, we will do it by showing that the mappings

$$
\hat{w} \mapsto\|\hat{\kappa} \hat{\nabla} \hat{w}\|_{0, \hat{K}}, \quad \hat{w} \mapsto\|\hat{\nabla} \hat{\bar{\kappa}} \hat{w}\|_{0, \widehat{K}}
$$

are norms on the corresponding factor spaces

$$
P_{r}(\widehat{M}) /\{\widehat{w}: \hat{\kappa} \hat{\nabla} \hat{w}=\mathbf{0}\} \quad \text { and } \quad P_{r}(\widehat{M}) /\{\hat{w}: \hat{\nabla} \hat{\bar{\kappa}} \hat{w}=\mathbf{0}\} .
$$

Let us assume that $\hat{\kappa} \hat{\nabla} \hat{w}=\mathbf{0}$. Then,

$$
\hat{\nabla} \hat{w}=\hat{\pi} \hat{\nabla} \hat{w} \in\left(P_{r-1}(\widehat{K})\right)^{d} \quad \Longrightarrow \quad \hat{w} \in P_{r}(\widehat{K}) \quad \Longrightarrow \quad \hat{I} \hat{w}=\hat{w} \quad \Longrightarrow \quad \hat{\nabla} \hat{\bar{\kappa}} \hat{w}=\mathbf{0} .
$$

Conversely, assuming $\hat{\nabla} \hat{\kappa} \hat{w}=\mathbf{0}$, we obtain

$$
\hat{w}=\hat{I} \hat{w}+\text { const. } \in P_{r}(\widehat{K}) \quad \Longrightarrow \quad \hat{\nabla} \hat{w}=\hat{\nabla} \hat{I} \hat{w} \in\left(P_{r-1}(\widehat{K})\right)^{d} \quad \Longrightarrow \quad \hat{\pi} \hat{\nabla} \hat{w}=\hat{\nabla} \hat{w} \quad \Longrightarrow \quad \hat{\kappa} \hat{\nabla} \hat{w}=\mathbf{0} .
$$

Thus, we conclude the existence of two constants $C_{3}$ and $C_{4}$ such that

$$
C_{3}\left\|\kappa_{h} \nabla w_{h}\right\|_{0, K} \leq\left\|\nabla \bar{\kappa}_{h} w_{h}\right\|_{0, M} \leq C_{4}\left\|\kappa_{h} \nabla w_{h}\right\|_{0, K} \quad \forall w_{h} \in Y_{h}, \quad \forall K \in \mathcal{T}_{h}
$$

and the stabilising terms $S_{h}^{3}$ and $S_{h}^{1}$ are spectrally equivalent.

Remark 5.6. For quadrilateral and hexahedral elements we do not have in general the spectral equivalence of the stabilising terms. As an example we consider the case $d=2, r=1$. In the two-level approach we have on the macro element $\widehat{M}=(-1,+1)^{2}$ for the function $\hat{w}(\hat{x})=\hat{x}_{1} \hat{x}_{2}$

$$
\hat{\nabla} \hat{w}-\hat{\pi} \hat{\nabla} \hat{w}=\hat{\nabla} \hat{w}=\left(\hat{x}_{2}, \hat{x}_{1}\right)^{T},
$$

but the Lagrange interpolant $\hat{I}$ in $Q_{1}(\widehat{M})$ leads to

$$
\hat{I} \hat{w}=\hat{w} \quad \Longrightarrow \quad \hat{\nabla}(\hat{w}-\hat{I} \hat{w})=(0,0)^{T} .
$$

The same argument holds for enriched approximation spaces $Y_{h}$ on a reference cell $\widehat{K}$.

\section{Conclusions}

We have investigated in this paper the local projection stabilisation for the Oseen equations on simplices, quadrilaterals, and hexahedra in a general manner.

Starting with an abstract framework, we have seen that three ingredients are essential for stable discretisations with optimal error estimates. The first two conditions are approximation properties of the approximation space $Y_{h}, c f$. Assumption A1, and the projection space $D_{h}, c f$. Assumption A2, respectively. These assumptions are fulfilled for a large class of spaces. The third condition requires the inf-sup stability of the local approximation space $Y_{h}(M)$ and the local projection space $D_{h}(M)$, cf. Assumption A3.

We have considered two different types of local projection schemes. The projection space in the two-level approach is based on a coarser macro triangulation while the projection space in the enrichment method is defined on the same mesh as the approximation space. The enrichment method is easier to implement and generates a stencil which is much more compact than the stencil for the two-level approach, $c f$. Remark 4.5. Moreover, one can construct an enrichment method on quadrilaterals and hexahedra such that the local approximation space contains compared to the usual $Q_{r}$-elements just $d$ functions more where $d$ is the space dimension. 
Finally, we have seen that the local projection scheme and the subgrid modelling introduced by Guermond result in the same discrete problems provided that piecewise linears on simplices are considered. Moreover, we have shown that the stabilisation terms of the local projection scheme and the subgrid modelling are spectrally equivalent for higher order elements on simplices. Unfortunately, this equivalence doesn't hold on quadrilaterals and hexahedra.

\section{REFERENCES}

[1] L. Alaoul and A. Ern, Nonconforming finite element methods with subgrid viscosity applied to advection-diffusion-reaction equations. Numer. Meth. Part. Diff. Equat. 22 (2006) 1106-1126.

[2] T. Apel, Anisotropic finite elements. Local estimates and applications. Advances in Numerical Mathematics. Teubner, Leipzig (1999).

[3] D.N. Arnold, D. Boffi and R.S. Falk, Approximation by quadrilateral finite elements. Math. Comput. 71 (2002) 909-922.

[4] R. Becker and M. Braack, A finite element pressure gradient stabilization for the Stokes equations based on local projections. Calcolo 38 (2001) 173-199.

[5] R. Becker and M. Braack, A two-level stabilization scheme for the Navier-Stokes equations, in Numerical mathematics and advanced applications, M. Feistauer et al. Eds., Berlin, Springer-Verlag (2004) 123-130.

[6] R. Becker and B. Vexler, Optimal control of the convection-diffusion equation using stabilized finite element methods. Numer. Math. 106 (2007) 349-367.

[7] M. Braack and E. Burman, Local projection stabilization for the Oseen problem and its interpretation as a variational multiscale method. SIAM J. Numer. Anal. 43 (2006) 2544-2566.

[8] M. Braack and T. Richter, Solutions of 3D Navier-Stokes benchmark problems with adaptive finite elements. Comput. Fluids 35 (2006) 372-392.

[9] M. Braack and T. Richter, Stabilized finite elements for 3D reactive flows. Int. J. Numer. Methods Fluids 51 (2006) $981-999$.

[10] M. Braack and T. Richter, Solving multidimensional reactive flow problems with adaptive finite elements, in Reactive Flows, Diffusion and Transport, W. Jäger, R. Rannacher and J. Warnatz Eds., Springer-Verlag (2007) 93-112.

[11] M. Braack, E. Burman, V. John and G. Lube, Stabilized finite element methods for the generalized Oseen problem. Comput. Methods Appl. Mech. Engrg. 196 (2007) 853-866.

[12] A.N. Brooks and T.J.R. Hughes, Streamline upwind/Petrov-Galerkin formulations for convection dominated flows with particular emphasis on the incompressible Navier-Stokes equations. Comput. Methods Appl. Mech. Engrg. 32 (1982) 199-259.

[13] P.G. Ciarlet, The finite element method for elliptic problems. SIAM (2002).

[14] P. Clément, Approximation by finite element functions using local regularization. RAIRO Anal. Numér. 9 (1975) 77-84.

[15] A. Ern and J.-L. Guermond, Theory and practice of finite elements, Applied Mathematical Sciences 159. Springer-Verlag, New York (2004).

[16] L.P. Franca and S.L. Frey, Stabilized finite element methods: II. The incompressible Navier-Stokes equations. Comput. Methods Appl. Mech. Engrg. 99 (1992) 209-233.

[17] T. Gelhard, G. Lube, M.A. Olshanskii and J.-H. Starcke, Stabilized finite element schemes with LBB-stable elements for incompressible flows. J. Comput. Appl. Math. 177 (2005) 243-267.

[18] V. Girault and P.-A. Raviart, Finite Element Methods for Navier-Stokes Equation, SCM 5. Springer-Verlag, Berlin (1986).

[19] J.-L. Guermond, Stabilization of Galerkin approximations of transport equations by subgrid modelling. ESAIM: M2AN 33 (1999) 1293-1316.

[20] J.-L. Guermond, Subgrid stabilization of Galerkin approximations of linear contraction semi-groups of class $C^{0}$. Comput. Visual. Sci. 2 (1999) 131-138.

[21] J.-L. Guermond, Subgrid stabilization of Galerkin approximations of linear contraction semi-groups of class $C^{0}$ in Hilbert spaces. Numer. Meth. Part. Diff. Equat. 17 (2001) 1-25.

[22] J.-L. Guermond, Subgrid stabilization of Galerkin approximations of linear monotone operators. IMA J. Numer. Anal. 21 (2001) 165-197.

[23] J.-L. Guermond, A. Marra and L. Quartapelle, Subgrid stabilized projection method for 2d unsteady flows at high Reynolds numbers. Comput. Methods Appl. Mech. Engrg. 195 (2006) 5857-5876.

[24] T.J.R. Hughes, Multiscale phenomena: Green's functions, the Dirichlet-to-Neumann formulation, subgrid scale models, bubbles and the origins of stabilized methods. Comput. Methods Appl. Mech. Engrg. 127 (1995) 387-401.

[25] T.J.R. Hughes and G. Sangalli, Variational multiscale analysis: Projection, optimization, the fine-scale Greens' function, and stabilized methods. USNCCM8, Austin (2005) 27-29.

[26] T.J.R. Hughes and G. Sangalli, Variational multiscale analysis: The fine-scale Green's function, projection, optimization, localization, and stabilized methods. SIAM J. Numer. Anal. 45 (2007) 539-367. 
[27] T.J.R. Hughes, L.P. Franca and M. Balestra, A new finite element formulation for computational fluid dynamics. V: Circumventing the Babuška-Brezzi condition: A stable Petrov-Galerkin formulation of the Stokes problem accomodating equal-order interpolations. Comput. Methods Appl. Mech. Engrg. 59 (1986) 85-99.

[28] V. John, On large eddy simulation and variational multiscale methods in the numerical simulation of turbulent flows. Appl. Math. 51 (2006) 321-353.

[29] V. John and S. Kaya, A finite element variational multiscale method for the Navier-Stokes equations. SIAM J. Sci. Comput. 26 (2006) 1485-1503.

[30] V. John, S. Kaya and W.J. Layton, A two-level variational multiscale method for convection-dominated convection-diffusion equations. Comput. Methods Appl. Mech. Engrg. 195 (2006) 4594-4603.

[31] S. Kaya and B. Rivière, A two-grid stabilization method for solving the steady-state Navier-Stokes equations. Numer. Meth. Part. Diff. Equat. 22 (2005) 728-743.

[32] G. Lube, Stabilized FEM for incompressible flow. Critical review and new trends, in European Conference on Computational Fluid Dynamics ECCOMAS CFD 2006, P. Wesseling, E. Onate and J. Périaux Eds., The Netherlands (2006) 1-20 TU Delft.

[33] G. Lube and G. Rapin, Residual-based stabilized higher-order FEM for a generalized Oseen problem. Math. Models Methods Appl. Sci. 16 (2006) 949-966.

[34] G. Matthies, Mapped finite elements on hexahedra. Necessary and sufficient conditions for optimal interpolation errors. Numer. Algorithms 27 (2001) 317-327.

[35] G. Matthies and G. Lube, On streamline-diffusion methods of inf-sup stable discretisations of the generalised Oseen problem. Preprint 2007-02, Institut für Numerische und Angewandte Mathematik, Georg-August-Universiät Göttingen (2007).

[36] G. Matthies and L. Tobiska, The inf-sup condition for the mapped $Q_{k}-P_{k-1}^{\text {disc }}$ element in arbitrary space dimension. Computing 69 (2002) 119-139.

[37] H.-G. Roos, M. Stynes and L. Tobiska, Numerical methods for singularly perturbed differential equations. Convection-diffusion and flow problems, SCM 24. Springer-Verlag, Berlin (1996).

[38] L. R. Scott and S. Zhang, Finite element interpolation of nonsmooth functions satisfying boundary conditions. Math. Comput. 54 (1990) 483-493.

[39] R. Stenberg, Analysis of mixed finite element methods for the Stokes problem: A unified approach. Math. Comput. 42 (1999) 9-23.

[40] L. Tobiska, Analysis of a new stabilized higher order finite element method for advection-diffusion equations. Comput. Methods Appl. Mech. Engrg. 196 (2006) 538-550.

[41] L. Tobiska and R. Verfürth, Analysis of a streamline diffusion finite element method for the Stokes and Navier-Stokes equation. SIAM J. Numer. Anal. 33 (1996) 107-127. 